\title{
Muséologies
}

Les cahiers d'études supérieures

\section{Des espaces monographiques au sein des collections muséales : déléguer ou produire une pensée et une pratique critiques de la collection?}

\section{Anne Bénichou}

Volume 5, numéro 1, automne 2010

URI : https://id.erudit.org/iderudit/1033522ar

DOI : https://doi.org/10.7202/1033522ar

Aller au sommaire du numéro

Éditeur(s)

Association Québécoise de Promotion des Recherches Étudiantes en Muséologie (AQPREM)

ISSN

1718-5181 (imprimé)

1929-7815 (numérique)

Découvrir la revue

Citer cet article

Bénichou, A. (2010). Des espaces monographiques au sein des collections muséales : déléguer ou produire une pensée et une pratique critiques de la collection ? Muséologies, 5(1), 40-83. https://doi.org/10.7202/1033522ar
Résumé de l'article

L'objectif principal de cet article est d'évaluer si l'acquisition d'espaces monographiques dédiés à des artistes permet de pallier certaines limites des modes de collection traditionnels des musées d'art moderne et contemporain. L'auteure distingue deux cas de figure. Dans le premier, le musée délègue entièrement à l'artiste la conception et le fonctionnement de son espace monographique. Celui-ci est dès lors traité comme une oeuvre autonome à inscrire dans la collection, sans forcément en redéfinir les modalités. Dans le deuxième, l'institution travaille en étroite collaboration avec l'artiste, l'espace monographique devenant un lieu d'expérimentation artistique et muséologique qui génère une pensée et des pratiques critiques de collection. 
Anne Bénichou

Des espaces monographiques au sein des collections muséales: déléguer ou produire une pensée et une pratique critiques de la collection?

L'objectif principal de cet article est d'évaluer si l'acquisition d'espaces monographiques dédiés à des artistes permet de pallier certaines limites des modes de collection traditionnels des musées d'art moderne et contemporain. L'auteure distingue deux cas de figure. Dans le premier, le musée délègue entièrement à l'artiste la conception et le fonctionnement de son espace monographique. Celui-ci est dès lors traité comme une œuvre autonome à inscrire dans la collection, sans forcément en redéfinir les modalités. Dans le deuxième, l'institution travaille en étroite collaboration avec l'artiste, l'espace monographique devenant un lieu d'expérimentation artistique et muséologique qui génère une pensée et des pratiques critiques de collection.

Anne Bénichou est professeure d'histoire et de théorie de l'art à l'Ecole des arts visuels et médiatiques de l'Université du Québec à Montréal. Ses travaux portent sur les archives, les formes mémorielles et les récits historiques issus des pratiques artistiques contemporaines et des institutions chargées de les préserver et de les diffuser. Elle s'intéresse également à la documentation et à la transmission des œuvres éphémères. Elle a publié en zolo aux Presses du réel l'ouvrage collectif Ouvrir le document. Enjeux et pratiques de la documentation dans les arts visuels contemporains. BENICHOU.ANNE@UQAM.CA 

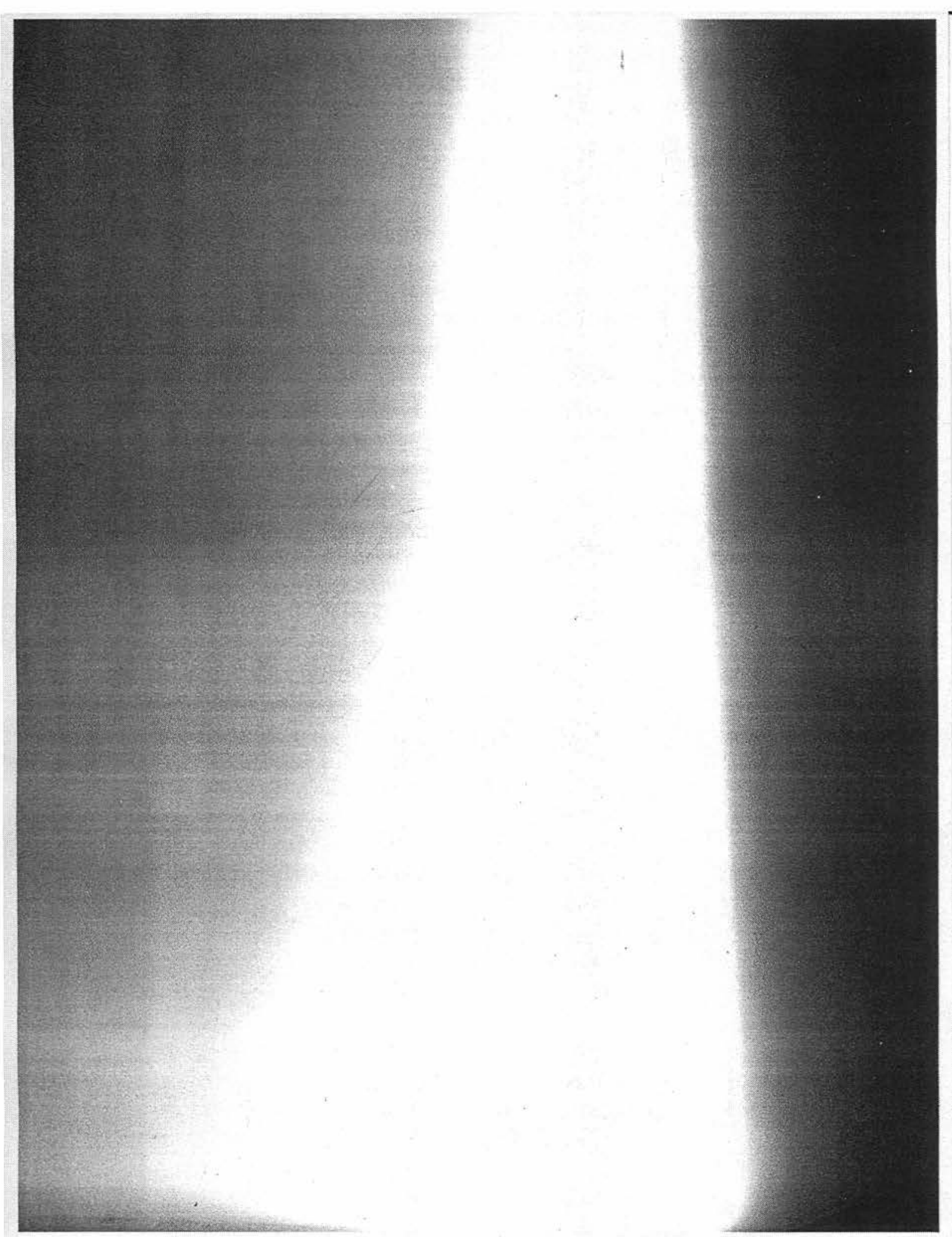
,

Ces formes artistiques qui sont apparues à la fin des années 1960 ont reçu diverses appellations. "Musée d’artistes", proposé par le commissaire Harald Szeemann en 1972 à l'occasion de documenta 5 (la documenta est une exposition d'art contemporain organisée à Kassel en Allemagne tous les cinq ans), a connu la plus grande fortune critique. Toutefois, la notion "d'espace monographique" que privilégie Christian Bernard, directeur du Musée d'art moderne et contem-; porain de Cenève, apparaît plus intéressante parce qu'elle désigne des objets à la fois artistiques (des installations prenant la forme d'expositions monographiques) et muséographiques (des expositions monographiques prenant valeur d'installations).
Au cours des dernières décennies du XXe siècle, sont apparus au sein des collections muséales d'art contemporain des objets que l'on pourrait qualifier d'espaces monographiques' parce qu'ils excèdent à plusieurs égards l'idée communément admise d'œuvre. Ils la débordent d'abord par leur échelle. Ils semblent relever plus d'un corpus d'œuvres que d'une seule œuvre. L'artiste y fait souvent une synthèse de travaux qui dans le passé avaient été conçus et présentés de façon autonome. Certains espaces monographiques prennent une dimension rétrospective indéniable, les artistes y menant une récapitulation de leur démarche. De plus, il est fréquent que ces espaces monographiques soient évolutifs. Leurs auteurs les développent durant de nombreuses années, parfois leur vie entière. Leur acquisition par un musée n'arrête pas forcément leur transformation. Certaines institutions la prévoient même et l'encadrent. Enfin, ces objets se distinguent par leur caractère "exposé" ou "installatif". Le spectateur ne sait pas toujours s'il doit les appréhender comme des installations ou des expositions. Cette ambiguïté est d'autant plus grande lorsque l'espace monographique accueille les œuvres d'autres créateurs, l'artiste jouant alors le rôle d'un conservateur ou d'un commissaire chargé de la mise en exposition d'un corpus de son choix dans un espace dont il a la responsabilité.

Ces pratiques artistiques qui sont apparues à la fin des années 1960 (bien qu'il y ait eu des précurseurs au cours de la modernité) procèdent d'un empiètement des artistes sur les responsabilités traditionnelles du conservateur. S'y joue une renégociation de leurs champs de compétences respectifs. Malgré les tensions que ces réglages ont parfois entraînées, les institutions muséales ont commencé à acquérir ces œuvres dès les années 1970, selon trois modalités. La plus courante consiste à appréhender l'espace monographique lorsque l'artiste y a mis un terme et qu'il en a arrêté les éléments constitutifs et le dispositif de présentation. Une deuxième modalité est d'acquérir l'espace monographique pendant qu'il est en cours de développement. Il s'intègre alors à la collection sous une forme évolutive dont les paramètres font souvent l'objet d'une entente ou d'un contrat entre l'artiste et l'institution. Plus 
récemment, certains musées commandent aux artistes des espaces monographiques pour leur collection, ceux-ci pouvant être fixés ou évolutifs.

Le statut de ces objets au sein des collections muséales varie considérablement. Dans la plupart des cas, ils sont considérés comme des œuvres à part entière sur lesquelles les artistes exercent pleinement leurs droits d'auteur. Comme pour une installation, les éléments qui les constituent sont en principe inaliénables et ne peuvent pas être exposés séparément, même si dans le passé ils ont été considérés comme des œuvres autonomes. Selon une logique inverse, d'autres espaces monographiques sont envisagés comme des ensembles composés d'éléments autonomes qui peuvent isolément tenir lieu d'œuvre à part entière. Quant à ceux qui accueillent les œuvres d'autres créateurs, leur statut est plus complexe. Ils fonctionnent selon une figure d'emboîtement, une œuvre qui en contient une autre ou plusieurs autres et, par conséquent, oscillent entre l'installation et l'exposition.

$\AA$ partir d'un corpus de sept cas choisis en raison de leur exemplarité des formes et des statuts d'espaces monographiques, de leurs modalités d'acquisition et des conceptions de la collection des institutions qui les ont acquises, il s'agira ici de comprendre ce phénomène de collectionnement d'espaces monographiques. Leur acquisition de plus en plus courante relève d'une tentative de pallier certaines apories des collections muséales d'art contemporain régulièrement dénoncées par les artistes depuis la création des musées : la collection par échantillonnage où l'œuvre est envisagée comme la synecdoque de l'ensemble de la pratique de l'artiste ou d'une période importante de son développement; la tendance à fixer les œuvres au moment de leur acquisition, privilégiant leur "valeur historique " au détriment de leur "valeur artistique " ${ }^{2}$; la mise en exposition des collections permanentes qui répond trop souvent à des récits historiques, chronologiques, stylistiques ou nationaux, jugés caducs, surtout depuis les débats sur le postmodernisme.
2

J'utilise tout au long de cet article les notions de "valeur historique» et de "valeur artistiquen dans le sens où l'entendent Françoise Choay et Aloïs Riegl. La "valeur historique" procède du savoir et de l'érudition et envisage les œuvres en tant que témoins de l'époque dont elles sont issues; elle la documente. La "valeur d'art " relève au contraire de l'expérience perceptuelle vécue des œuvres qui n'implique pas forcément le recours à un savoir historique. L'œuvre s'adresse à notre sensibilité artistique, à notre "vouloir d'art». (CHOAY, Françoise. L'allégorie du patrimoine. Paris: Seuil, 1992; RIECL, Aloìs. Le culte moderne des monuments. Son essence et sa genèse. Paris: Seuil, 1984.) 
3

La notion d'authenticité est comprise ici dans le sens d'une conformité matérielle à un original. (CHOAY, Françoise. "Sept propositions sur le concept d'authenticité et son usage dans les pratiques du patrimoine historiquen. Conférence de Nara sur l'authenticité dans le cadre de la Convention du Patrimoine Mondial. UNESCO, 1995, p. 101-120.)
Les espaces monographiques permettent aux artistes de mettre en scène l'ensemble de leur œuvre, ou tout au moins un corpus important et non quelques échantillons, de formuler des principes d'intelligibilité de leur travail et d'articuler des récits différents de ceux que les institutions produisent. Enfin, la variabilité de certains d'entre eux remet en question les valeurs de pérennité et d'authenticité $^{3}$ traditionnellement attachées à la collection. A travers leurs espaces monographiques, les artistes pensent la question de la pérennité de leur œuvre et inventent de nouvelles façons d'en assurer la transmission. Quel impact cette réflexion a-t-elle sur les musées qui acquièrent ces objets?

Deux positions institutionnelles assez différentes se dégagent. Pour certains musées, l'acquisition d'espaces monographiques permet d'intégrer des formes critiques de collection sans avoir l'obligation de les produire et de les mettre en pratique, selon un principe de délégation à l'artiste et de repliement de l'espace monographique à l'intérieur du périmètre de l'œuvre qu'il constitue. Chez d'autres, on constate des formes de collaboration, de coproduction et parfois une appropriation de certaines stratégies mises en œuvre par les artistes dans leurs espaces monographiques. L'institution ne se contente dès lors plus de déléguer la production d'une forme critique de collection à l'artiste, mais contribue à la produire en collaboration avec lui. L'espace monographique y est conçu comme un espace d'expérimentation artistique et muséologique, élaboré par l'artiste et l'institution, qui génère de nouvelles formes de pérennisation des œuvres et des récits hétérodoxes de l'art contemporain.

\section{La délégation à l'artiste et la clôture de l'espace monographique}

Un premier corpus d'espaces monographiques, constitué du Magasin de Ben de Ben Vautier au Musée national d'art moderne (MNAM), de Pour mémoire de Christian Boltanski au CAPC (Centre des arts plastiques contemporains) musée d'art contemporain de Bordeaux, et de Pretty Much Every Film and Video From About 1992 de Douglas Gordon acquis par le Museum of Modern Art of San Francisco 
(SFMOMA) et le Musée d'art moderne de la ville de Paris, procède d'une délégation à l'artiste d'une forme critique de collection, celui-ci exerçant ses prérogatives au sein d'un espace monographique qui est conçu comme une œuvre à part entière et sur laquelle il exerce pleinement ses droits. Dans le cas de Ben Vautier, le musée délègue entièrement à l'artiste "l'implémentation 4" du Magasin dans l'espace muséal, y compris la mise en œuvre de sa valeur documentaire et de sa dimension historique. Une période d'ajustement assez longue est prévue dès l'acquisition au cours de laquelle l'artiste peut apporter les modifications qu'il juge nécessaires. Pour mémoire de Christian Boltanski a présenté davantage de difficultés puisque l'artiste préconisait pour son espace monographique une sortie du lieu muséal. Les compromis trouvés consistent en deux remaniements successifs, entièrement assumés par l'artiste, qui entraînent toutefois un retournement du rapport que l'espace monographique devait initialement entretenir avec le musée. Pretty Much Every Film procède d'un partage des responsabilités entre les musées qui en ont fait l'acquisition et l'artiste. Les premiers assument la préservation de l'œuvre, selon l'acception traditionnelle du musée comme dépositaire. Douglas Gordon ou ses assistants orchestrent les réexpositions successives selon des configurations à chaque fois différentes, augmentées de nouveaux éléments.

\section{Une délégation entière à l'artiste}

Le Magasin de Ben, 1958-1973, de l'artiste Fluxus Ben Vautier, a été acquis en 1975 par le MNAM, alors que Pontus Hulten en était le directeur (ill. 1). Il s'agit de la reconstitution de la boutique que Ben avait tenue à Nice de 1958 à 1973 et qui faisait office de commerce, d'œuvre d'art totale et d'espace de diffusion artistique. L'artiste y vendait des disques et des livres d'occasion; il y accumula une quantité d'objets hétéroclites qu'il utilisa pour transformer l'espace en sculpture in progress. À la mezzanine, une galerie successivement dénommée Laboratoire 32, Galerie Ben doute de tout et Centre d'art total présentait des expositions d'artistes d'horizons divers: les nouveaux réalistes, Support Surface, Fluxus, le Gutai, etc. Sur le trottoir, devant la devanture, se tenaient
4

J'utilise tout au long de cet article ce terme dans le sens où l'entend Nelson COODMAN (L'art en théorie et en action. Paris: Éditions de l'Éclat. 1996): l'implémentation d'une œuvre consiste en un ensemble de procédures entreprises pour faire fonctionner une œuvre. 


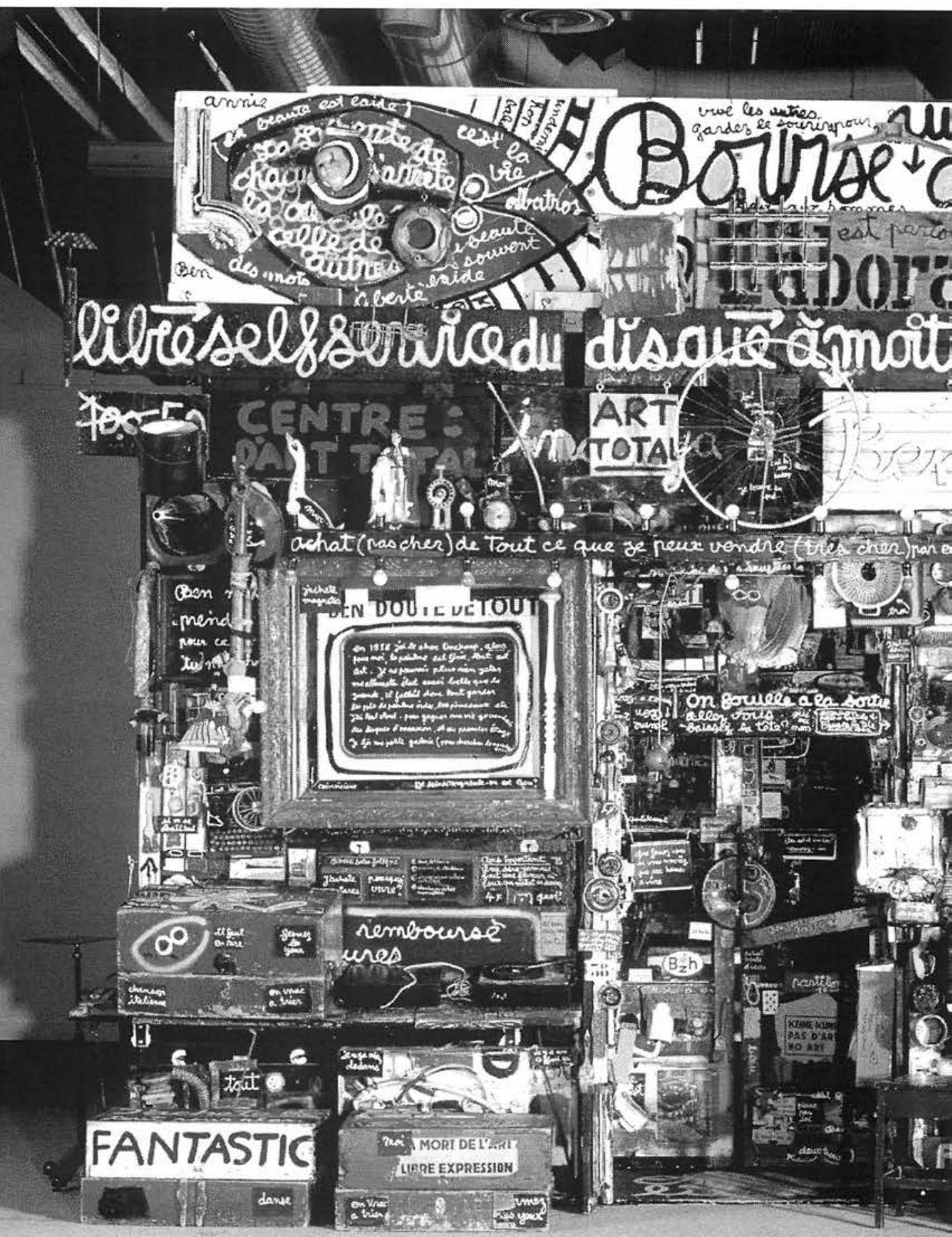




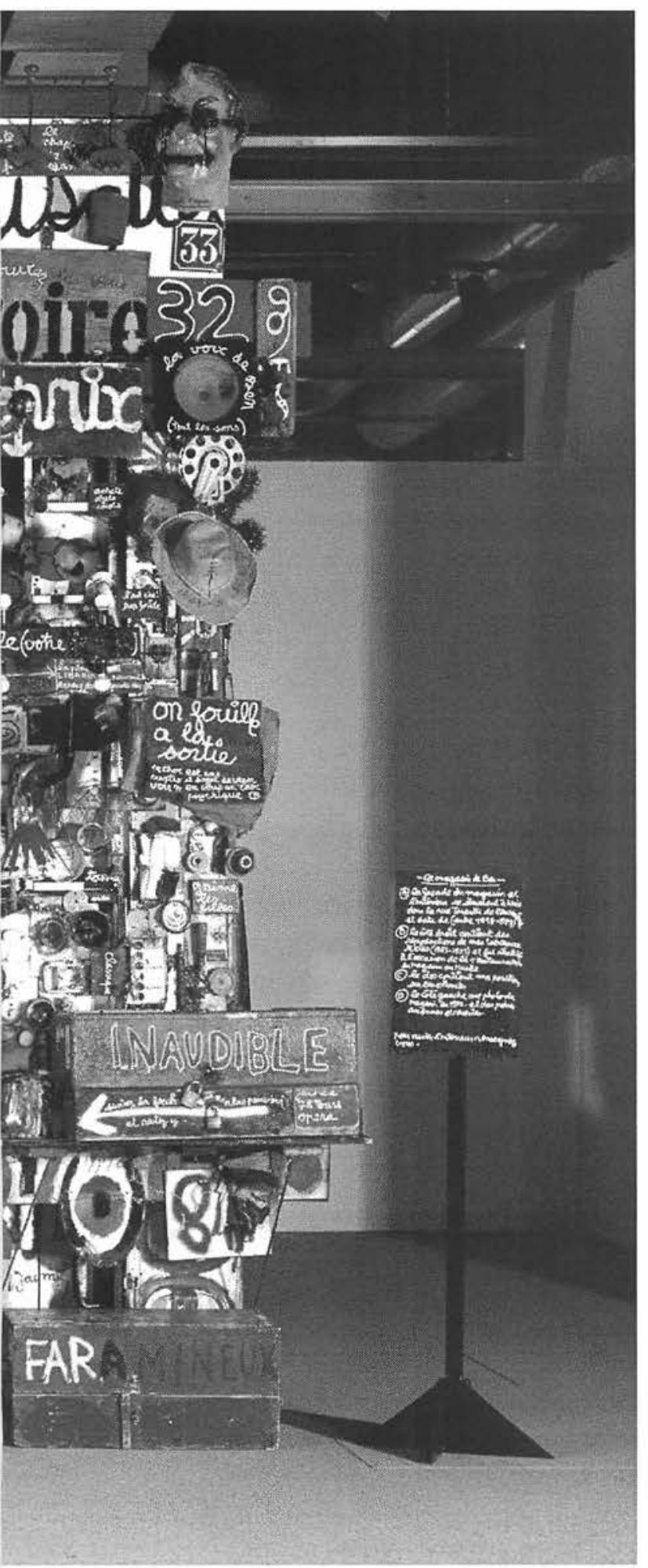

\section{Illustration 1}

Ben (Vautier, Benjamin) (1935-), Le Magasin de Ben. 1958-1973. Matériaux divers. $350 \times 500 \times 350 \mathrm{~cm}$. Musée national d'Art Moderne, Centre Pompidou, Paris, France, AM 1975-185. [Photo: Philippe Migeat], crédit photographique: CNAC/MNAM/Dist. Réunion des Musées Nationaux/Art Resource, New York. @ ARS, NY 
5

VAUTIER, Ben. Cahier des charges. Dossier de l'œuvre. Documentation des Collections contemporaines, Musée national d'art moderne, Paris, n.d., n.p.

6

Id.

7

DUPLAIX, Sophie (dir.). Collection art contemporain: la collection du Centre Pompidou. Paris: Musée national d'art moderne, Centre Pompidou, 2007 , p. 60

8

Correspondance entre Ben Vautier et Alice Brutin, 25 novembre 1975 et 8 décembre 1975. Dossier de l'œuvre. Documentation des Collections contemporaines, Musée national d'art moderne, Paris: "Le magasin de Ben [...] est constitué d'éléments faisant effectivement partie de l'agencement intérieur et extérieur du magasin tel qu'il était entre 1958 et 1972 dans la rue Tondotti de l'Escarène à Nice." régulièrement des happenings et des actions artistiques. Ben y avait également installé le "bureau » de la revue Tout qu'il publiait alors.

Lorsqu'il décida de fermer le magasin, Ben envisagea immédiatement de le reconstituer, afin de pouvoir l'exposer. Dans ses propos, il insiste sur la valeur historique et documentaire du Magasin, ainsi que sur sa dimension holistique:

Le magasin est la pièce la plus représentative de ma période 1958 à 1963. C'est elle qui représente le mieux la période d'appropriation et l'esprit d'art total. Le magasin en fait est une œuvre qui court sur 10 ans et qui m'a entouré et a entouré toutes mes activités pendant cette période ${ }^{5}$.

La reconstitution muséologique de la boutique est conçue comme un tout qui doit être exposé dans son entièreté, aucun élément ne pouvant en être soustrait: "En aucune manière, écrit Ben, le magasin ne peut être démantelé ou vendu ou exposé en pièces séparées ${ }^{6}$."

Le défi que pose l'intégration de cette œuvre à l'institution muséale est que, en dépit de sa muséification et de ses dimensions historiques et documentaires pleinement assumées par l'artiste, elle suscite une expérience esthétique qui rend compte de la fusion de l'art et de la vie dont elle procédait. Dès le début, il fut prévu que Ben y apporterait des ajustements et des modifications, afin de "lui donner une vie propre dans ce nouveau contexte $" 7$. Durant quinze ans, les remaniements, les ajouts et les réactivations successifs furent effectués par l'artiste, à l'occasion du montage de l'œuvre pour des expositions ou de nouveaux accrochages de la collection permanente. À la fin des années 1990, le MNAM cessa de faire appel à l'artiste, mettant ainsi fin aux transformations.

Les modifications apportées par Ben procèdent d'une tension entre les valeurs historiques et artistiques. Les objets et les différents éléments provenaient du magasin originel de Nice et une certaine valeur d'authenticité y était donc attachée ${ }^{8}$. L'entente passée entre le directeur Pontus Hulten et Ben prévoyait l'acquisition des archives de l'artiste relatives à l'histoire de sa boutique: une photographie de 
très grand format représentant la devanture en 1972, un film documentant les activités qui s'y sont tenues de 1958 à 1963 , ainsi que divers imprimés issus de cette période. Il était prévu que ces archives accompagnent la mise en vue du Magasin $^{9}$ et non pas qu'elles soient destinées à un dossier documentaire, selon l'usage courant. Les imprimés furent disposés sur la paroi extérieure arrière, donnant un aperçu des expositions et des activités éditoriales que menait Ben dans son magasin. La photographie de la façade, d'abord exposée sur un mur adjacent, fut intégrée au magasin et accrochée à la paroi extérieure gauche, accompagnée d'une plaque portant le nom de la rue où il se trouvait à Nice. Au fil des modifications, cette documentation riche en information historique fut de plus en plus intégrée aux diverses interventions plastiques que Ben réalisa sur les parois extérieures, l'artiste cherchant à imbriquer les espaces de l'œuvre et de l'archive qui traditionnellement sont séparés.

Pour conserver l'aspect vivant du magasin et en proposer une expérience esthétique pertinente, Ben avait à l'origine prévu que les spectateurs puissent circuler à l'intérieur assez librement. Il avait également envisagé la construction d'un socle de même dimension qu'un trottoir, afin d'évoquer l'espace de la rue. Pour continuer à faire vivre cet espace, il orchestra diverses formes d'actualisation. Lors de la première présentation de l'œuvre au Centre Pompidou en 1977, il organisa une exposition des artistes Cutai, rappelant l'importante activité de diffusion artistique du magasin à Nice. En 1994, à l'occasion de l'exposition Hors limites qui était consacrée au happening et à la performance, il réalisa une performance le soir du vernissage et laissa sur place, pour la durée de l'exposition, tous les accessoires qu'il avait utilisés. Il s'agissait de redonner à cet espace la dimension expérimentale qu'il avait à Nice en y organisant une action artistique nouvelle, ne faisant pas partie d'un répertoire. Enfin, à même la matière textuelle de l'œuvre, Ben eut le souci de dater ses différents ajouts, afin de donner à voir le processus de réactualisation qui soustend le magasin. On lit ainsi, sous un texte inscrit sur la paroi gauche: «texte apposé sur le côté gauche du magasin
9

BRUTIN, Alice. Dossier d'acquisition de l'œuvre. 18 juin 1975. Documentation des Collections contemporaines, Musée national d'art moderne, Paris. 
à l'occasion de l'exposition Hors limites 1994 ". Un autre texte portant sur le multiculturalisme est daté de 1985.

Les interventions successives que Ben réalisa sur les parois extérieures du magasin démontrent également un souci de concevoir la représentation la plus complète de sa démarche. Le.panneau droit constitue à lui seul une exposition des écritures peintes de Ben portant sur le sens et la fonction de l'art; tandis que sur la paroi arrière un texte dénonce l'ethnocentrisme culturel du milieu de l'art et plaide en faveur du multiculturalisme, une rhétorique et une thématique récurrentes chez Ben. L'intérieur du magasin quant à lui procède de l'appropriation et du recyclage d'objets courants. Ce sont presque toutes les facettes de la démarche de l'artiste qui sont ainsi convoquées.

Tout au long de ce processus, le musée délègue à l'artiste l'entière responsabilité de son espace monographique, de ses modifications et de sa mise en perspective historique. A l'instar des autres artistes Fluxus, Ben a pris très tôt en charge la construction du discours historique sur son œuvre et la constitution des archives de sa pratique et de celle de ses collègues, selon une approche très artistique de la représentation historique: confusion des espaces de l'œuvre et de l'archive, traitement plastique de l'archive, tensions entre authenticité et réactualisation, fantasme d'une œuvre holistique. Cette production du discours historique inhérente à la démarche des artistes Fluxus explique en partie pourquoi Hulten a octroyé une telle marge de liberté à Ben.

Si le Magasin met en jeu une réflexion sur la préservation et des formes hétérodoxes de traitement de l'archive en regard de celles qui sont couramment en usage dans les musées, il n'oblige en rien l'institution à revoir sa conception de la collection et ses modes de présentation. Constitué en œuvre qui a son autonomie, le Magasin peut sans difficulté intégrer un récit traditionnel de l'art contemporain, comme le parcours chronologique. Il introduit de surcroît une distance critique à l'égard de ce récit, qui peut dès lors être perçu comme une forme d'autocritique institutionnelle. 
De plus, l'acquisition du Magasin s'inscrit dans une politique d'acquisition d'œuvres monumentales que le MNAM amorça en 1973 et qui constitue l'une des spécificités de sa collection $^{\circ}$. Ces œuvres permettaient une représentation significative de certains artistes, sans pour autant opter pour un collectionnement de séries monographiques qui, aux yeux des directeurs successifs, aurait contrevenu au mandat d'un musée national". La dimension holistique du Magasin, de plus en plus affirmée au fil des modifications, s'arrimait parfaitement à cette approche de la collection.

\section{Des compromis dans le respect de la pleine autorité de l'artiste}

Conçu en 1974 dans la perspective d'une critique de la collection muséale, l'espace monographique de Christian Boltanski au CAPC musée d'art contemporain de Bordeaux, bien qu'il procède de compromis successifs, n'ébranle pas le principe de la pleine autorité de l'artiste sur son espace monographique. Pour mémoire est constitué d'un corpus de documents et de fragments issus des premières activités artistiques pour la plupart éphémères que Boltanski a menées entre 1966 et 1973: pages de ses livres d'artistes, photographies, lettres, menus objets, dessins (ill. 2). Ils sont accrochés sur les murs ou déposés dans des vitrines, de façon aléatoire, sans respecter les séries ni les corpus dont ils sont issus, dans un petit espace clos de l'Entrepôt Lainé qu'occupe le CAPC. Une lucarne percée dans la porte permet aux visiteurs d'apercevoir l'intérieur de la cellule et son contenu de façon partielle, sans qu'ils puissent y pénétrer. L'œuvre se découvre au hasard de la visite. Aucun fléchage ne mène à son emplacement. Pour entrer à l'intérieur, il faut en faire la demande et prendre rendezvous. Cette installation qui mise sur une certaine invisibilité et illisibilité a été réalisée par l'artiste en 2005, soit 31 ans après qu'il ait donné à l'institution les documents et les artefacts qui la composent. Pour mémoire est le fruit d'un compromis et d'une renégociation des modes d'articulation de l'entreprise d'automuséification de Boltanski à l'institution. Il convient de relater l'histoire assez complexe de cette œuvre pour en saisir les enjeux.
10

"Des hommes et une collection". Le Magazine CNAC, 15 mai - 15 juin 1992, p. 11.

11

Sur cet aspect de la collection du MNAM : Catherine David en entretien avec Chantal Pontbriand. "Le musée national d'art moderne. Du musée soixante-huitard au musée néo-classique". Parachute, $n^{2} 46$, printemps 1987, p. 30-34; LÉVESQUE, France. La collection muséale d'art contemporain aux limites du pluralisme et du syncrétisme identitaire. Thèse de doctorat en histoire de l'art, Montréal (Québec): Université du Québec à Montréal, 2003. p. 52-56. 


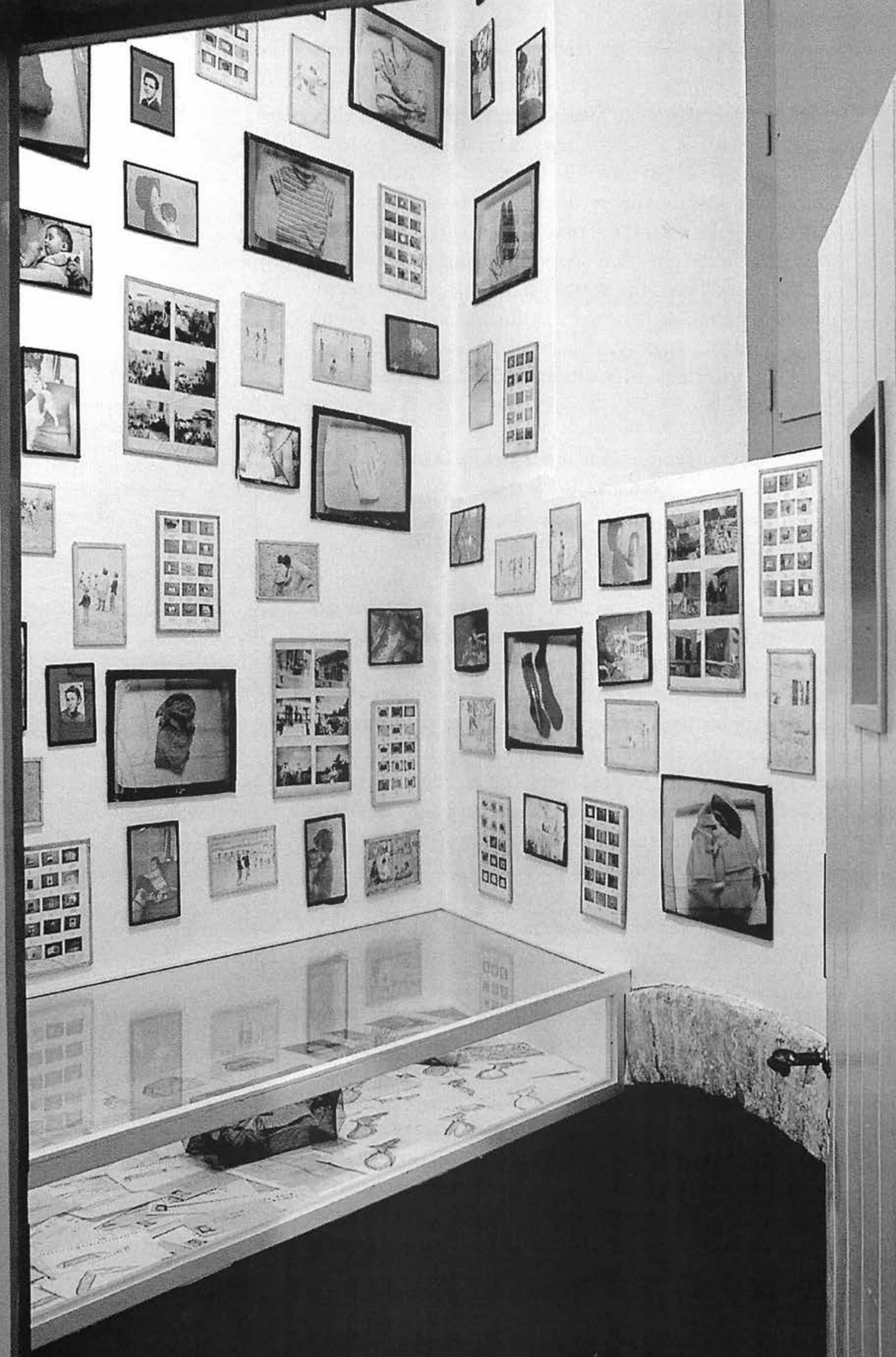




\section{Illustration 2}

Christian Boltanski, Pour Mémoire, 1974, CACP musée d'art contemporain de Bordeaux, inv.1990-04, [Photo: F. Delpech]

\section{Illustration 3}

Christian Boltanski, Les Réserves du Musée Christian Boltanski, 1974, CAPC musée d'art contemporain de Bordeaux, inv. 1990-04,

[Photo: F. Delpech]

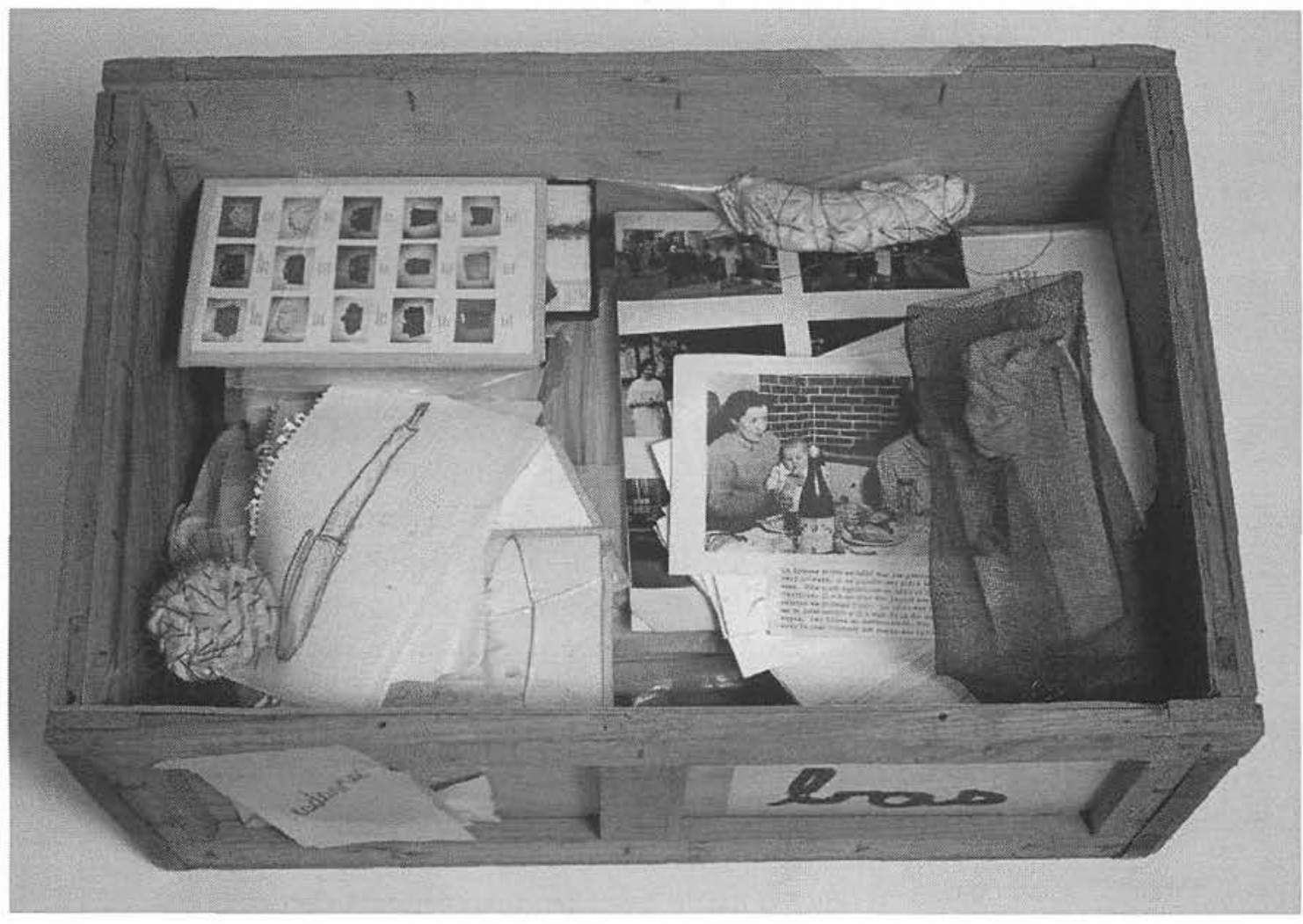


Boltanski avait exposé ce corpus de documents en 1974 au CAPC - qui était alors un centre d'exposition sans collection - à l'occasion de l'exposition collective itinérante Pour mémoires, organisée par le directeur Jean-Louis Froment et Suzanne Pagé, responsable de L'ARC (animation, recherche, création). L'intervention de Boltanski intitulée Musée Christian Boltanski consistait à présenter aux murs et sous vitrines les résidus de ses activités artistiques, en mimant les dispositifs de présentation des musées. Elle s'inscrivait dans l'entreprise d'automuséification, largement critique, que l'artiste avait amorcée deux années auparavant. Par l'appropriation d'opérations muséographiques, Boltanski cherchait à souligner le caractère mortifère des entreprises de préservation, à procéder à un effacement de l'artiste et à un glissement des beaux-arts vers l'anthropologie. Ces muséographies de soi étaient empreintes d'un fort sentiment de perte ; elles instauraient une sorte d'anonymat à cause de la banalité des objets qui étaient présentés et semblaient relever du musée ethnographique ou archéologique plus que de celui des beaux-arts.

À la fin de l'exposition Pour mémoires, Boltanski fit don du corpus au CAPC, demandant que cette collection soit présentée en tant que Musée Christian Boltanski dans un espace situé hors des locaux de l'institution, idéalement une maison individuelle. Le cadeau était à bien des égards empoisonné. Le CAPC n'avait pas à l'époque le mandat de constituer une collection. L'artiste ne donna aucune instruction quant aux modalités de présentation du corpus. Au moment du démontage de sa pièce, il se contenta d'entasser les objets et les documents dans une caisse de bois empruntée à des fins pratiques à l'installation d'un autre artiste de l'exposition, Jean-Paul Thibeau. Enfin, le projet de créer un Musée Christian Boltanski permanent hors d'une institution consacrée à l'art contemporain avait jusqu'alors échoué, malgré différentes tentatives soutenues par des personnalités du milieu artistique. Ainsi, en 1974, lorsque Boltanski réalisa au Centre national d'art contemporain (CNAC) l'Inventaire des objets ayant appartenu à une femme de Bois-Colombes, une installation présentant sous vitrine les objets quotidiens d'une femme de la banlieue parisienne, il demanda à Blaise 
Cautier et à Alfred Pacquement'12 de faire acquérir l'œuvre, en tant que Musée Christian Boltanski, par un musée de province, si possible consacré à l'archéologie'?

Au CAPC, l'acquisition du Musée Christian Boltanski ne fut officiellement entérinée qu'en 1990 , soit quatorze ans après le dépôt du corpus. Son intégration à l'institution qui était désormais chargée de constituer une collection s'accompagna d'une modification de son titre par Les Réserves du Musée Christian Boltanski, indiquant qu'il s'agissait davantage d'un dépôt, d'un stock dans l'attente d'un usage. Au même moment, plusieurs œuvres de Boltanski furent acquises par l'institution bordelaise, constituant un corpus imposant axé sur les années 1970. La politique d'acquisition que le directeur Jean-Louis Froment venait tout juste de définir privilégiait les séries monographiques de quelques artistes de son choix, en opposition aux modes plus traditionnels de collection relevant d'un échantillonnage des principales tendances. Dans cette perspective, les Réserves du Musée Christian Boltanski présentait un double intérêt. On y retrouvait des fragments des versions imprimées de plusieurs des installations nouvellement acquises, mettant donc en évidence les stratégies de reprise et le travail d'édition de l'artiste. Elles contenaient les traces d'autres travaux de la même époque. Elles formalisaient la façon dont Boltanski envisageait la mémoire de son œuvre.

Toutefois, l'exigence de l'artiste d'exposer l'œuvre dans un lieu extérieur au musée constituait un défi que l'institution ne parvint pas à relever. De son acquisition officielle en 1990 à son remaniement en 2005, les artefacts furent donc présentés dans la boîte de Thibeau, après entente avec les deux artistes ${ }^{14}$ (ill. 3). Ce mode de présentation qui procède de l'impossibilité d'implémenter l'œuvre selon les prescriptions originelles de l'artiste relève d'un remaniement (puisqu'il reconfigure la mise en vue de 1974) pleinement assumé par Boltanski et qui tend à renforcer le sentiment de perte que suscite l'œuvre, L'entassement des objets et des documents dans la boîte rendait la plupart d'entre eux invisibles. L'absence de toute organisation sérielle significative infirmait les tentatives d'interprétation de ces
12

En 1974, Blaise Gautier était directeur du Centre national d'art contemporain et Alfred Pacquement était responsable des expositions auprès du directeur du MNAM. 13 Procès-verbal de la réunion du 24 avril 1974 et lettres de Blaise Gautier du 6 juin 1974. Documents conservés dans le dossier d'archives de l'exposition Boltanski - Monory. Archives du Centre Pompidou, Paris.

14

Les Réserves du Musée Christian Bolatnski furent présentées lors des expositions Collections pour une région: Même si c'est la nuit en 1994 et Entre le ciel et l'eau en 1995, toutes deux organisées au CAPC musée d'art contemporain de Bordeaux. 
15

Anne Cadenet, en entretien téléphonique avec l'auteure le 26 juillet 2010. artefacts. Il s'agissait davantage de les appréhender comme des restes dont le sens était à jamais perdu.

Avec les années, et en raison de la pauvreté et de la précarité des matériaux sciemment voulues par l'artiste, plusieurs éléments se dégradèrent : la colle des cadres en ruban adhésif coula; plusieurs vitres se brisèrent, etc. A la fin des années 1990, cet état de conservation conférait à l'œuvre des allures de ruines: les ruines de l'œuvre de Christian Boltanski. Cette détérioration matérielle n'était certes pas en contradiction avec le projet conceptuel de l'artiste, mais, me semble-t-il, portait atteinte à l'intégrité matérielle de l'œuvre et à la qualité de l'expérience que le spectateur en faisait.

En 2005, après de nouvelles tentatives infructueuses pour trouver un espace extérieur, le CAPC proposa à l'artiste une solution de compromis qui consistait à investir un local technique exigu du bâtiment qui n'avait jamais été utilisé pour exposer des œuvres. Les Réserves du Musée Christian Boltanski y seraient installées en permanence. L'artiste accepta la proposition et eut carte blanche quant à la disposition des artefacts dans l'espace. La chargée de conservation, Anne Cadenet, qui travailla avec Boltanski, envisage cette nouvelle transformation comme un autre remaniement au cours duquel l'artiste "exerça pleinement son droit moral sur son œuvre», le rôle de l'institution étant de "l'accompagner et de le soutenir" dans cette démarche ${ }^{15}$. Le CAPC procéda également à la remise en état des éléments endommagés, tout en respectant l'esthétique pauvre de la pièce. Le titre fut encore modifié. Pour mémoire était la reprise au singulier de l'exposition de Froment et de Pagé. Le cartel apposé à l'entrée du local indique bien cette référence historique:

Euvre installée par Christian Boltanski le 4 janvier 2005. Le titre Pour mémoire, choisi par l'artiste pour cette installation in situ est un hommage rendu au titre de l'exposition de Jean-Louis Froment qui présenta cette œuvre dans sa toute première version en 1974 lors de l'exposition Pour mémoires au CAPC. 
Ce nouveau remaniement provoque un retournement de l'articulation de l'œuvre au musée. Alors que l'artiste souhaitait échapper à l'espace institutionnel, en tant qu'œuvre in situ, Pour mémoire en est indissociable. De surcroît, l'œuvre de Boltanski est désormais porteuse d'une histoire institutionnelle, celle des débuts du CAPC, très marquante en France. On l'aura compris, alors que Boltanski avait établi une relation critique avec les musées au début des années 1970 en proposant des projets qui en éprouvaient les limites, en 2005, après une carrière pleine de reconnaissances officielles et dans un contexte idéologique totalement différent, l'œuvre s'inscrit pleinement dans l'institution. Elle en devient sa crypte destinée aux reliques de son histoire.

\section{Un partage des responsabilités à la faveur de l'artiste}

Si l'œuvre de Douglas Cordon semble a priori reposer sur un mode plus collaboratif, le partage des responsabilités que l'artiste instaure maintient le musée dans un rôle traditionnel de dépositaire. Comme son titre l'indique non sans une pointe d'humour, Pretty Much Every Film and Video From About 1992 consiste à exposer tous les films et les vidéos que l'artiste a réalisés depuis 1992 sur des moniteurs disposés dans une seule salle (ill. 4). Présentée pour la première fois en 1999 à la galerie Foskal à Varsovie, elle est depuis régulièrement exposée selon le principe d'accroissement indiqué par le titre, le nombre de films et de vidéos étant passé d'une trentaine à une cinquantaine en dix ans. Cette démarche récapitulative constamment mise à jour joue avec l'idée de "l'œuvre complète" qu'Olivier Corpet décrit comme un : " [moment crucial] de récapitulation, de réorganisation, de reclassement. Moment de construction d'une nouvelle forme, d'une nouvelle logique de l'œuvre - et donc, ipso facto, moment d'une nouvelle lecture et d'une nouvelle réception de celle-ci. ${ }^{16}$

Gordon toutefois confère un caractère provisoire et instable à son "œuvre complète». D'exposition en exposition, elle est constamment mise à jour et entièrement reconfigurée.
16

CORPET, Olivier. "L'archive-œuvre". in. POINSOT, Jean-Marc (dir.). Les artistes contemporains et l'archive. Interrogation sur le sens du temps et de la mémoire à l'ère de la numérisation. Rennes: Presses universitaires de Rennes, 2004, p. 43. 


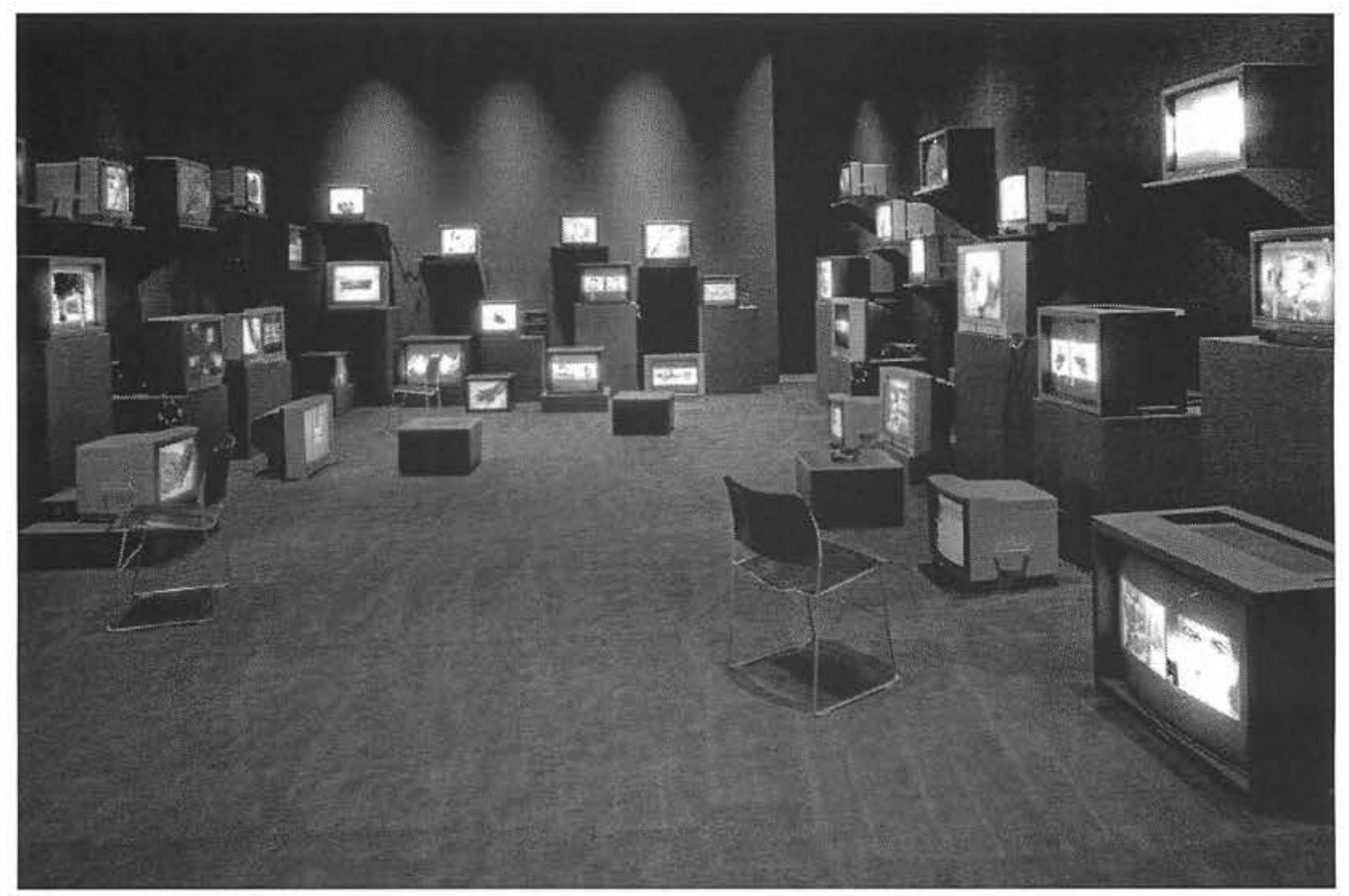

\section{Illustration 4}

Douglas Gordon, Pretty much every film and video work from about 1992 until now. To be seen on monitors, some with headphones, others run silently, and all simultaneously, 1992 à aujourd'hui, installation vidéographique, dimensions variables, Collection SFMOMA, achat avec le soutien de Roselyne Chroman Swig et du Accessions Committee Fund, - Douglas Gordon, courtesy Gagosian Gallery, New York 
Aucun plan de montage n'est arrêté et aucun appareil de visionnement n'accompagne l'œuvre. L'artiste souhaite que chaque institution présente la pièce avec les lecteurs de DVD et les moniteurs qu'elle possède dans ses réserves et ses locaux techniques, à l'exception des appareils trop récents. Ils sont disposés de façon aléatoire dans une salle, posés à terre, sur des étagères, des socles, des caisses, des tables, également trouvés sur place. L'expérience doit évoquer au spectateur le visionnement des films à la télévision, dans un espace domestique ${ }^{17}$. De plus, l'accrochage doit éviter toute forme de catégorisation et d'ordonnancement du corpus pour privilégier la dispersion et le mélange des procédés, des thèmes, des périodes de production. Pretty Much n'en propose pas moins un regard intéressant sur l'œuvre de Gordon. Elle constitue l'unique occasion d'appréhender la presque totalité du corpus filmique et vidéographique de l'artiste. Chaque spectateur est libre de créer ses propres associations entre les œuvres, sans qu'aucune lecture ne lui soit imposée. Enfin, le dispositif spatial est si différent de celui des installations vidéographiques dans lesquelles ces images sont habituellement projetées que l'expérience des œuvres s'en trouve renouvelée.

Malgré le caractère évolutif de l'œuvre qui ne prendra fin qu'avec l'arrêt de la production artistique de Cordon, deux musées en ont fait l'acquisition : le Musée d'art moderne de la ville de Paris, en 2004, et le San Francisco Museum of Modern Art (SFMOMA), en 2008. On saisit la pertinence d'intégrer Pretty Much à une collection : elle propose un regard sur l'ensemble de l'œuvre de Gordon ; elle constitue un complément judicieux à l'acquisition de ses installations vidéographiques; elle relève d'une conception de la rétrospective qu'aucun commissaire ou historien d'art n'aurait pu proposer. Le défi qui se posait toutefois aux deux institutions était d'encadrer le caractère évolutif de l'œuvre dans un contexte de collection muséale. La solution trouvée consiste en un partage des responsabilités : les musées agissent en tant que dépositaires chargés de la préservation; l'artiste prend en charge les réactualisations.
17

Ce souci de ramener l'expérience de visionnement des œuvres à une expérience télévisuelle est d'autant plus intéressant qu'un bon nombre de films repris par Gordon nous sont connus par le biais de la télévision. 
18

Courriel de Tanya Zimbardo, conservatrice adjointe des arts médiatiques, San Francisco Museum of Modern Art, $7^{\text {er juin } 2010 .}$

19

En tant que dépositaire d'une œuvre à composantes technologiques, le SFMOMA a réalisé en collaboration avec l'artiste un plan de migration technologique de l'œuvre, pour le futur.
Les deux versions acquises couvrent une période de temps déterminée, une décennie (1992-2002) pour l'œuvre parisienne, deux décennies pour l'cuvre états-unienne (19922012). Alors que l'institution française a acheté une version arrêtée de l'œuvre comptant une trentaine de films et de vidéos, l'institution américaine a opté pour un corpus ouvert sur une période de quatre ans au cours de laquelle l'artiste s'est engagé à verser ses nouvelles réalisations. Les deux versions se distinguent également par leur dispositif de présentation. En France, il s'agit de la version blanche, présentée dans une pièce peinte en blanc, à San Francisco de la version noire, installée dans une salle entièrement noire. Ces différences confèrent une singularité à chacune des œuvres, qui sont dès lors considérées comme des exemplaires uniques.

Ces acquisitions ne fixent toutefois pas les modalités de présentation. A chaque exposition, l'œuvre est reconfigurée avec le matériel de visionnement et le mobilier disponibles sur place, sans plan de montage. L'artiste ou son assistant participe à l'accrochage en étroite collaboration avec le commissaire et l'équipe technique de l'institution hôte. Gordon de surcroît fournit les copies de DVD par l'intermédiaire de sa galerie. Ainsi, en 2008, lors de l'exposition collective Retrospective à la galerie Cagosian à New York, Pretty Much était identifiée comme étant l'exemplaire unique du SFMOMA, bien que les DVD aient été fourni par la galerie et que l'artiste ait fait l'accrochage avec le personnel de la Gagosian en utilisant l'équipement et le mobilier trouvés sur place ${ }^{18}$. Autrement dit, sur un plan matériel, aucun élément constitutif de l'exemplaire du SFMOMA n'était présent. Toutefois, conformément aux spécificités du dispositif d'exposition de cet exemplaire, la salle était entièrement noire.

Dans le partage des responsabilités, le musée s'en tient strictement à son rôle de dépositaire. C'est d'ailleurs en termes de "repository " que Gordon parle de Pretty Much ${ }^{19}$. Au sein de la collection, l'œuvre, malgré son médium reproductible et son caractère évolutif, est ramenée vers une forme d'unicité (par l'attribution d'une couleur singulière) 
et de fixité (par l'arrêt de l'augmentation du corpus). Mais elle continue à vivre, à se déployer, à se modifier dans d'autres espaces. La production des nouvelles occurrences reste l'entière responsabilité de l'artiste qui ne se prive pas de prendre parfois les plus grandes libertés. Par exemple, en 2009, il a réédité Pretty Much pour l'exposition Running Time. Artist Films in Scotland 1960 to Now organisée par les National Galleries of Scotland. Présenté sur un seul écran selon une succession d'extraits de 20 secondes des films qui le composent, l'ensemble d'une durée de 25 minutes est intitulé Approximately 20 Seconds Worth of Pretty Much Every Video and Film Work From About 1992 Until Now...20.

Par le biais de leurs espaces monographiques, Ben Vautier, Christian Boltanski et Douglas Gordon formalisent des conceptions de la pérennisation de leur œuvre. Tous trois optent pour une forme holistique qui permet de montrer sinon la totalité de l'œuvre, du moins une représentation significative. Selon des approches très différentes, ils rejettent une conception de la collection basée sur les valeurs de pérennité et sur la fixation des œuvres : Ben multiplie les formes de réactualisation; Gordon instaure une dialectique entre l'unicité et la reproductibilité, entre la fixité et la réactualisation; Boltanski met en scène la ruine de son œuvre, à travers des remaniements successifs. Ils ébranlent également la valeur historique et documentaire qui est traditionnellement attachée à l'œuvre collectionnée, sans forcément la rejeter. Ben est celui qui y reste le plus attaché tout en cherchant à établir une étroite đialectique entre valeur historique et valeur artistique ; Boltanski la met en pièce ; Gordon cherche à multiplier les relectures individuelles sans privilégier de récits consensuels.

Les espaces monographiques présentent un double intérêt pour les institutions qui en font l'acquisition. Ils leur permettent d'intégrer à leur collection une représentation significative de la démarche d'un artiste, ainsi qu'un discours critique sur la pérennisation et la transmission des œuvres. A la fin des années 1970 et au début des années 1980 , de nombreuses institutions se mirent à privilégier la constitution de séries monographiques pour
20

Cette nouvelle version de l'œuvre est d'autant plus pertinente que sa forme installative avait été présentée dans la même institution trois ans auparavant, lors de l'exposition Douglas Gordon Superhumanatural. 
21

France LEVESQUE analyse ce phénomène (op. cit., p. 41-70) leur collection. Plusieurs directeurs de musées préférèrent cibler un petit nombre d'artistes et développer des corpus significatifs de leur démarche au lieu de fonctionner par échantillonnage d'artistes et d'œuvres jugés les plus représentatifs d'une période ${ }^{21}$. Cette nouvelle tendance était directement liée à la rupture épistémologique qui s'effectuait dans la discipline de l'histoire de l'art et qui mettait en pièce le "grand récit " moderniste qui sous-tendait les principales collections occidentales d'art moderne et contemporain. L'individualisation des collections autour de quelques artistes et à partir du point de vue d'une personnalité, en général le directeur du musée, fut l'une des avenues fortement privilégiées au moment du «virage postmoderne" de la muséologie de l'art contemporain. Les espaces monographiques que créent les artistes répondent très bien à ces nouvelles orientations. Ils permettent aux musées qui ont adopté cette forme de collection de consolider les corpus qu'ils ont constitués. Il en est ainsi de Pour mémoire au CAPC musée d'art contemporain de Bordeaux. Pour les autres institutions qui, pour diverses raisons, n'ont pas opté pour ce virage, ils permettent de pallier les limites des corpus constitués. L'acquisition du Magasin de Ben par le MNAM s'inscrit dans une telle logique.

Parce qu'ils proposent souvent des conceptions critiques de la collection institutionnelle et de ses modes de pérennisation, les espaces monographiques permettent également aux musées qui en font l'acquisition d'inscrire une forme d'autocritique à même leur pratique de collection, selon une position qui consiste à être à la fois le sujet et l'objet de la critique. Toutefois, dans le cas des trois espaces monographiques vus précédemment, on constate une délégation complète à l'artiste de la production de cette réflexion critique sur la collection. Les espaces monographiques sont conçus comme des œuvres, des installations. Conformément à la tendance dominante de la muséologie de l'art contemporain, ils sont collectionnés et exposés selon une acception de l'intégrité de l'œuvre qui repose presque exclusivement sur l'intentionnalité de l'artiste, le discours prescriptif de ce dernier primant sur celui des professionnels des institutions. Or, ce repliement de l'espace 
monographique sur l'autorité de son créateur au nom du droit moral qu'il exerce sur son œuvre semble affaiblir la portée critique de ces œuvres parce qu'il légitime une position de retrait de l'institution qui peut dès lors faire l'économie de repenser ses conceptions et ses pratiques de la collection ${ }^{22}$. Pontus Hulten délègue à Ben l'entière responsabilité de l'implémentation du Magasin ; Anne Cadenet conçoit son rôle de conservatrice comme une accompagnatrice de l'artiste au moment du remaniement de Pour mémoire; Gordon garde la responsabilité exclusive des réexpositions de Pretty Much après l'acquisition de deux exemplaires de l'œuvre.

\section{L'espace monographique collaboratif, une expérimentation artistique et muséologique}

Le deuxième corpus d'espaces monographiques, constitué du Discours des éléments du collectif $\mathrm{BGL}^{23}$ au Musée des beaux-arts du Canada (MBAC), de l'Atelier d'aujourd'hui de Cérard Collin-Thiébaut et de l'inventaire 2 de Claude Rutault, tous deux au Musée d'art moderne et contemporain de Cenève (Mamco), ainsi que du Container zéro de Jean-Pierre Raynaud au MNAM, fonctionne différemment. BGL, Collin-Thiébaut et Rutault partagent davantage leur auctorialité avec les professionnels des musées et, corollairement, ces derniers désirent développer des approches plus expérimentales et inventives de la collection. Ces espaces monographiques génèrent donc de nouvelles formes et pratiques de collection, leur dimension critique étant effective. L'efficience des deux premiers repose en partie sur l'ambiguïté de leur statut. Bien que le Discours des éléments soit une installation dont les modalités de présentation sont fixées, BCL accorde au musée acquéreur la possibilité d'exposer de façon autonome les fragments d'œuvres antérieures qui la composent, obligeant ainsi les professionnels de l'institution à imaginer d'autres configurations et d'autres récits. Selon une proposition inverse, L'Atelier d'aujourd'hui est une scénographie conçue en collaboration par l'artiste et le conservateur qui prend toutefois avec le temps valeur d'installation. Composée d'une cinquantaine d'œuvres
22

Mary Anne STANISZEWSKI (The Power of Display. A History of Exhibition Installations at the Museum of Modern Art. Cambridge, London: The MIT Press, 2001) a montré un phénomène semblable en regard de I'histoire des expositions du MoMA. A compter de la fin des années 1960, les artistes prennent souvent en charge le dispositif d'exposition et l'appareil interprétatif de leurs œuvres, légitimant une position de retrait de l'institution.

23

Collectif québécois regroupant trois artistes dont les initiales forment le sigle BCL: Jasmin Bilodeau, Sébastien Giguère et Nicolas Laverdière. 
autonomes, cultivant l'ambiguïté entre l'installation et la scénographie d'exposition, elle est régulièrement modifiée et devient un véritable terrain d'exploration muséologique et artistique. Les espaces monographiques de Rutault et de Raynaud ont quant à eux indéniablement le statut d'œuvre d'art, tout en constituant un espace d'exposition dans lequel l'œuvre d'autres artistes peut être présentée. Mais alors que Rutault laisse aux professionnels du musée une très grande liberté pour investir son espace et y concevoir des expositions, Raynaud s'octroie progressivement l'entière responsabilité de la programmation et tend à freiner les formes de collaboration.

\section{L'espace monographique en pièces détachées}

Créée en 2006, l'installation le Discours des éléments a la forme d'un entrepôt dans lequel gît sur des étagères un véritable bric-à-brac: des cadeaux emballés, un orignal empaillé, des pots de peinture, une moto accidentée, etc. Plusieurs de ces artefacts sont les fragments d'œuvres et de performances antérieures du collectif. On y retrouve des restes de treize œuvres qui ont été réalisées au cours des dix années d'existence de BGL, ainsi que des matériaux destinés au travail artistique. Parmi ces rayonnages encombrés, deux entrées sont camouflées. Un trou dans le mur qui semble avoir été causé par un incendie mène à une salle sombre au milieu de laquelle trône une voiture. Dans son coffre, le spectateur découvre le faux foyer qui pourrait bien être la cause de tout ce dégât. Un pan de mur qui pivote s'ouvre sur une salle lumineuse dans laquelle une boule de miroirs sur une balançoire à bascule projette ses reflets sur les murs, engendrant une véritable vision cosmique. Le discours des éléments peut être interprété comme une allégorie joyeuse de la création (l'informe, les matériaux bruts, la création de mondes, la destruction, le recyclage) et de ses lieux (l'atelier, la réserve).

Lors de son acquisition en 2007 par le MBAC, les modalités de présentation de l'œuvre sont rigoureusement fixées. Le musée a établi un cahier des charges et a réalisé une véritable cartographie photographique de la pièce. Seule une 
adaptation mineure aux dimensions des espaces d'exposition est autorisée par les artistes. Toutefois, Le discours des êléments est une œuvre en pièces détachées. Les artistes accordent au musée la possibilité d'exposer de façon autonome huit des treize œuvres recyclées dans l'installation. Dans une lettre à la conservatrice, le collectif précise: " Pour ce qui est de la douzaine d'œuvres 'importantes' contenues dans notre proposition [...], [certaines] peuvent être présentées de manière autonome, tandis que d'autres sont des restants qui témoignent d'interventions antérieures " ${ }^{24}$. Un document retrace également l'historique de chacune des œuvres et les différents contextes dans lesquels elles ont été présentées. Dans le catalogue informatisé de la collection du musée, un numéro d'inventaire, un titre et une date de production différents sont attribués à chacune des composantes. Le Discours des éléments établit ainsi un jeu de miroir habile avec l'institution qui la collectionne: c'est une réserve d'œuvres en attente d'être réexposées.

La réexposition de ces œuvres ne va pas de soi, car BCL les a présentées au fil des années selon des modalités très différentes et n'a cessé de les recycler d'une intervention artistique à l'autre. Les professionnels du musée devront non seulement réactualiser ces œuvres, mais ils auront aussi à les inscrire dans d'autres configurations, d'autres récits, et pourront même imaginer d'autres espaces monographiques. Cette marge de liberté très grande octroyée au musée constitue l'un des arguments que la conservatrice Josée Drouin-Brisebois avance pour justifier l'acquisition de l'œuvre:

Avec Le discours des éléments, BGL renforce la notion de mutabilité et d'instabilité des œuvres d'art. Cette œuvre polymorphe remet en question le statut d'une œuvre d'art et son contexte original. Certains des éléments autonomes peuvent être présentés comme des œuvres individuelles selon différents contextes. Les artistes donnent ainsi à l'institution la possibilité de disséquer l'installation, d'isoler certains des éléments qui ont leur propre finalité, afin qu'ils entrent en discours et se combinent à d'autres œuvres et espaces du musée. Alors que plusieurs institutions cherchent à conserver des fragments d'installation afin de montrer une trace de l'œuvre, l'acquisition de l'installation
24

Lettre de BGL à la conservatrice Josée Drouin-Brisebois. Archives de la collection, Musée des beaux-arts du Canada, n.d. 
25

DROUIN-BRISEBOIS, Josée. Pour la collection d'art contemporain canadien. Document de travail, Archives de la collection, Musée des beaux-arts du Canada, $7^{\text {er }}$ mai 2007, n.p. 26

Sur la genèse de L'Atelier d'aujourd'hui, voir l'entretien avec Christian Bernard dans le présent numéro de Muséologies. majeure Le discours des éléments permettrait de préserver non seulement une pièce composée de plusieurs incarnations autonomes de leur travail, mais une œuvre qui intègre l'histoire de sa production ${ }^{25}$.

L'Atelier d'aujourd'hui de Gérard Collin-Thiébaut, issu d'une commande du directeur du Mamco Christian Bernard à l'artiste, pose également la question de l'entité et du statut de l'espace monographique, mais selon une logique inverse puisqu'il consiste en une scénographie d'exposition conçue en collaboration par l'artiste et le directeur ${ }^{26}$. Elle emprunte à la fois les formes d'un atelier dans lequel l'artiste est invité à travailler épisodiquement et de la pièce d'un appartement bourgeois. Deux tables de travail sont installées au centre, dédiées à des travaux en cours de réalisation. Au mur, sont accrochées un nombre imposant d'œuvres de l'artiste qui pour la plupart appartiennent à la collection du Mamco, certaines ayant été acquises, d'autres déposées par l'artiste ou par des collectionneurs privés. Elles sont autonomes et peuvent être exposées ailleurs dans le musée ou prêtées à d'autres institutions. Les œuvres et leur accrochage sont régulièrement modifiés, tandis que la scénographie, en clin d'œil à l'univers bourgeois, devient de plus en plus cossue : lustre, moulure, plinthes, tapis, mobilier sont progressivement ajoutés. Peu à peu, ce dispositif d'exposition prend les allures d'une installation, qui plus est, dotée d'un titre. Les cartels qui identifiaient chacune des œuvres ont disparu afin de privilégier l'ensemble plutôt que les éléments constitutifs. La saturation de l'espace de plus en plus prononcée rend indissociables les œuvres de la scénographie. En 2005, lors de l'exposition L'ceuvre en programme organisée par le CAPC musée d'art contemporain de Bordeaux, l'ensemble de l'Atelier d'aujourd'hui, telle une installation, fut envoyé à Bordeaux où une salle aux mêmes proportions que celle du Mamco fut construite. L'Atelier d'aujourd'hui existe donc selon deux modalités : un corpus d'œuvres autonomes; un ensemble soigneusement scénographié qui avec le temps et les usages qui en sont faits prend valeur d'installation. 


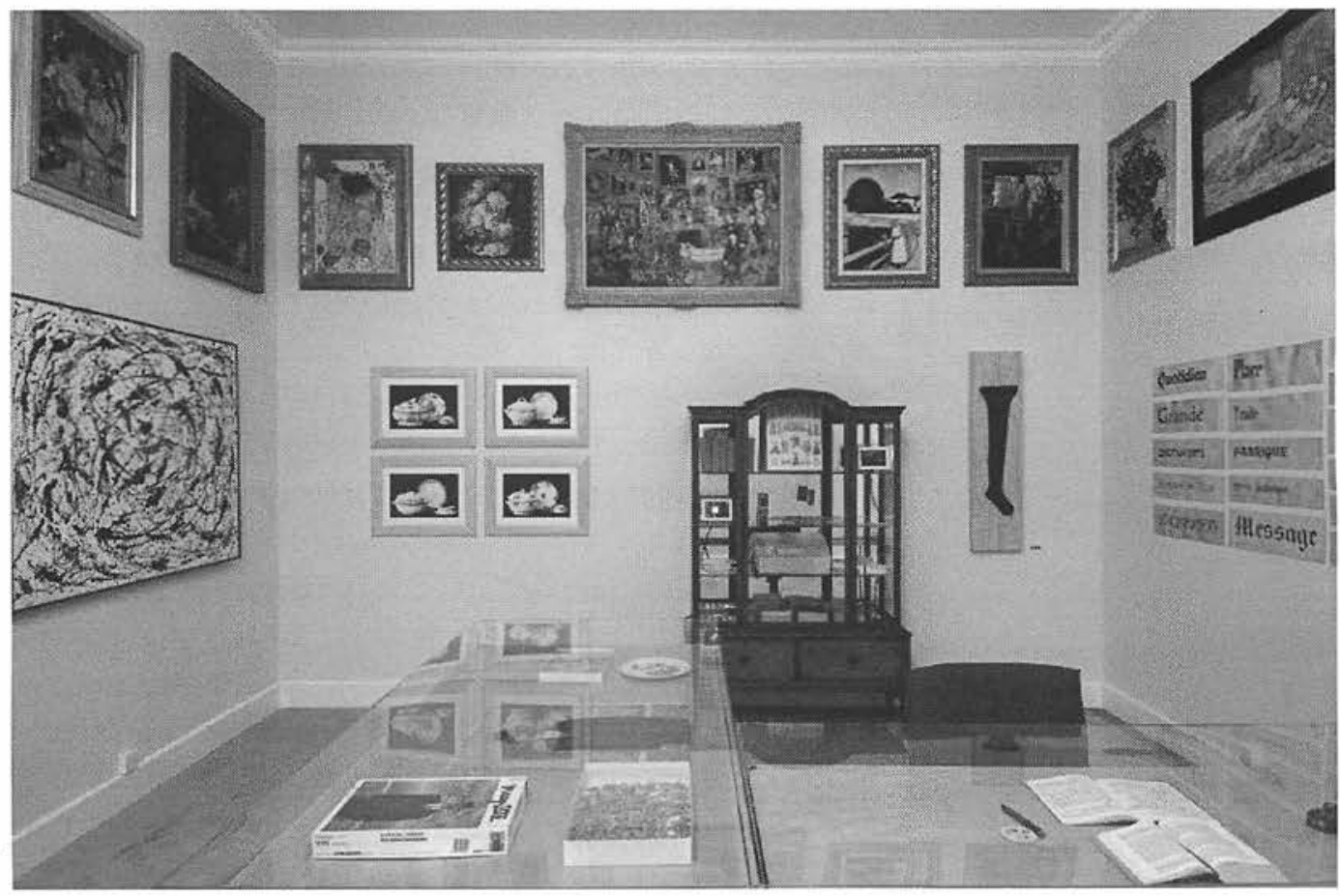

\section{Illustration 5}

Gérard Collin-Thiébaut, L'atelier d'aujourd'hui, 1994. Espace régulièrement actualisé par l'artiste et

mobilier (vitrine, table, lustre...). $400 \times 500 \times 500 \mathrm{~cm}$. Coll. Mamco, (- Mamco, Genève, [Photo: I.

Kalkkinen] 
Alors que BGL signe le dispositif installatif du Discours des éléments et en assume l'entière responsabilité sans délégation, L'Atelier d'aujourd'hui procède incontestablement d'une cocréation de l'espace monographique. Doit-on en déduire que le professionnel du musée empiète ici sur le territoire de l'artiste? L'implication de Bernard est d'ordre muséologique. Il met en place un dispositif qui permet d'éclairer une œuvre qui ne se laisse pas appréhender facilement. L'Atelier d'aujourd'hui regroupe des extraits des principales séries que Collin-Thiébaut mène simultanément sur de longues périodes et qui relèvent d'une démarche d'appropriation, déclinée selon différentes modalités : des reprises d'œuvres de l'histoire de l'art et de la littérature, des appropriations d'images diffusées dans les médias et la culture populaires, etc. Ces travaux procèdent d'actions qui confèrent au geste créatif une certaine trivialité : copier, reproduire, colorier, compléter des puzzles, découper des images, composer des rébus, etc. D'un point de vue visuel, l'artiste joue sur le registre de la déception. Il ne cherche pas à susciter un "plaisir de l'œil". La scénographie de L'Atelier d'aujourd'hui rend intelligible la logique sérielle de la démarche de Collin-Thiébaut. La forme de l'appartement réfère aux méthodes de travail de l'artiste qui, dans sa maisonatelier d'Ornans, consacre chacune des pièces à une série en cours. Enfin, par la coquetterie de plus en plus affirmée du décor, elle pallie la pauvreté visuelle sciemment voulue par l'artiste.

\section{Lorsqu'une œuvre en contient une autre}

Les espaces monographiques de Claude Rutault et de JeanPierre Raynaud, tout en ayant le statut d'œuvre d'art, sont conçus comme des espaces d'exposition pouvant recevoir l'œuvre d'autres artistes. Alors que Rutault en délègue la programmation aux professionnels du musée, Raynaud en conserve le contrôle. La définition/méthode 170 encore intitulée inventaire 2 procède d'une commande du Mamco à Rutault : une salle permanente rendant compte de son travail. L'artiste a proposé un inventaire de l'ensemble des énoncés programmatiques qui règlent les modalités de production, de diffusion et de documentation de son cuvre. Ces protocoles 
qu'il appelle les définitions/méthodes ou $\mathrm{d} / \mathrm{m}$ sont destinés aux preneurs en charge de ses œuvres, en l'occurrence les collectionneurs, qu'ils soient individuels ou institutionnels, qui ont la responsabilité de la réalisation matérielle de l'œuvre et de toutes les facettes de sa socialisation. La d/m 170 s'énonce de la façon suivante:

stock de toiles égal au nombre de définitions/méthodes écrites au jour de l'installation.

toutes les toiles, numérotées au dos selon l'ordre de rédaction des $\mathrm{d} / \mathrm{m}$ sont identiques.

la présentation se fait dans un lieu aménagé à cet effet :

1. les toiles sont rangées verticalement sur des étagères. la visibilité est maximale.

2. la présentation a lieu dans un espace fermé. l'œuvre n'est visible que de l'extérieur. un côté au moins de la salle est vitré.

3. l'aménagement est conçu de façon à pouvoir recevoir de nouvelles toiles et à s'adapter en fonction de circonstances particulières ou par rapport à d'autres $\mathrm{d} / \mathrm{m}$ actualisées ailleurs.

PEINTURE :

les toiles sont rangées d'après leur numéro sur les étagères. la toile est blanche si la $\mathrm{d} / \mathrm{m}$ à laquelle elle correspond a été actualisée, sinon elle est laissée brute. chaque année à la date anniversaire de l'installation, l'œuvre est mise à jour. des toiles sont ajoutées pour les nouvelles $d / m$ et celles qui ont été actualisées sont peintes en blanc.

il est possible d'extraire une toile, et de la présenter peinte de la même couleur que le mur, à la condition qu'elle corresponde à une $\mathrm{d} / \mathrm{m}$ installée à ce moment-là [soit parce qu'elle a été prise en charge, soit parce qu'elle figure dans une exposition].

INFORMATION :

une tablette intérieure accueille un certain nombre d'informations lisibles de l'extérieur: les données nécessaires à la compréhension de l'œuvre. l'adresse ou l'exposition à laquelle renvoie la toile présentée, l'information TRAVAUX PUBLICS, liste de toutes les œuvres prises en charge et les possibilités de visite..., le tout mis à jour régulièrement.

ARCHIVES :

le preneur en charge s'engage à conserver la totalité des informations concernant l'œuvre, documents que claude Rutault peut exploiter par la suite pour une publication ${ }^{27}$.
27

RUTAULT, Claude. «d/m 17o. inventaire 2". définitions/méthodes, le livre 1973-2000. Paris, Tours : productions Flammarion 4 G CCC tours, 2000 p. 1231. Voir aussi <http://www. mamco.ch/collections/rutault. html> (consulté le 7 septembre 2010). 
28

RUTAULT, Claude. "d/m 274. ce qu'il ne faut plus faire, 1997". définitions/ méthodes, le livre 1973-2000, op. cit., p. 1433.
En 1997, et selon une stratégie de retouche et de réécriture qui lui est familière, Rutault rédige $1 \mathrm{a} \mathrm{d} / \mathrm{m} 274$ ce qu'il ne faut plus faire, venant modifier la précédente en préconisant la soustraction d'un certain nombre de $d / m$ qui ne pourront désormais plus être actualisées. En voici une transcription partielle:

œuvre composée de toiles correspondant dans inventaire 2, d/m 170 (mamco, genève), aux $d / m$ supprimées, c'est-àdire non réalisables à partir de cette publication. en octobre 1997 ces toiles sont au nombre de 29 .

la liste ci-jointe n'est pas définitive, soit que d'autres viennent la rejoindre soit que certaines soient refondues d'une façon ou d'une autre dans le corpus.

toutes ces toiles sont peintes de façon uniforme d'une même couleur. le choix de cette couleur, variable d'une actualisation à l'autre, est laissé à la liberté d'expression du preneur en charge ${ }^{28}$.

Conformément aux prescriptions de l'artiste, le Mamco a aménagé en 1994 une salle fermée et vitrée munie d'étagères permettant d'entreposer les toiles correspondant à l'ensemble des $d / m$ existantes. Par la suite, et conformément à la d/m 274, 29 toiles furent peintes de couleur orange.

Les prescriptions de Rutault préconisent donc une mutabilité de l'inventaire inđiquant une résistance de la part de l'artiste à une représentation pérenne de son œuvre. L'inventaire est constamment à poursuivre, à corriger, à actualiser et à réactualiser. Elles prévoient également une délégation importante à l'institution. Les professionnels du musée prennent en charge sa réalisation matérielle, sa mise à jour, sa documentation, ainsi que l'actualisation de certaines $\mathrm{d} / \mathrm{m}$.

Tout en se conformant aux prescriptions de l'artiste, Christian Bernard transforme l'inventaire 2 en véritable espace d'exposition dans lequel d'autres œuvres de Rutault sont actualisées ainsi que les œuvres d'autres artistes, selon la logique d'une œuvre qui en contient une autre. En 2000 , s'y tient l'actualisation de la 176 internationale $d / m$, 
un ensemble de calicots portant, dans diverses langues, l'inscription :

\section{À VENDRE \\ Toiles tendues sur châssis}

\section{À PEINDRE}

De la même couleur que le mur sur lequel elles doivent être accrochées. S'adresser ici [suit un numéro de téléphone]. (ill. 6)

En 2001, toujours selon le même principe d'emboîtement, la $\mathrm{d} / \mathrm{m} 131$ entourant le tableau est actualisée dans l'espace de l'inventaire 2. Son énoncé se lit ainsi :

Un mur sur lequel est accroché un tableau. Un certain nombre de toiles l'entourant. Elles sont peintes de la même couleur que le mur. Une ou deux toiles sont identiques au tableau. Les autres, plus petites ou plus grandes, sont de dimensions proches. Ni le nombre ni l'accrochage ne sont déterminés à l'avance et peuvent toujours donner lieu à remords. Rien n'est fixe d'une réalisation à l'autre. L'œuvre peut être réalisée avec plusieurs tableaux sur un même mur à la condition que cette idée d'entourer le tableau avec des toiles peintes de la même couleur que le mur reste évidente et chaque tableau individualisé.

Le nombre de réalisations n'est pas limité ${ }^{29}$. (ill. 7)

Sur le mur du fond peint en rouge, quatre petits monochromes d'Allan McCollum de la série Colored Surrogates sont alignés et entourés de toiles monochromes rouges; sur le mur de droite de couleur bleue, des tableaux de Stephen Prina issus de la série Monochrome Painting et des monochromes bleus sont disposés selon le même principe. Tout en suivant les règles prescrites par l'artiste, Bernard intervient pleinement à titre de commissaire. Il organise, dans le périmètre de l'œuvre de Rutault, une exposition sur les pratiques picturales actuelles qui revisitent la tradition du monochrome. Les trois artistes, tout en ayant des parcours et des esthétiques différentes, ont été marqués par l'art conceptuel, ses interrogations sur la nature de l'œuvre d'art, sur ses conditions de production et de diffusion, prémices à partir desquelles ils abordent la tradition de la peinture abstraite monochromatique. Cette microexposition organisée dans les murs de l'inventaire 2 propose un
29

RUTAULT, Claude. “d/m 131. entourant le tableau, 1981". définitions/ méthodes, le livre 1973-2000, op. cit., p. 1174 


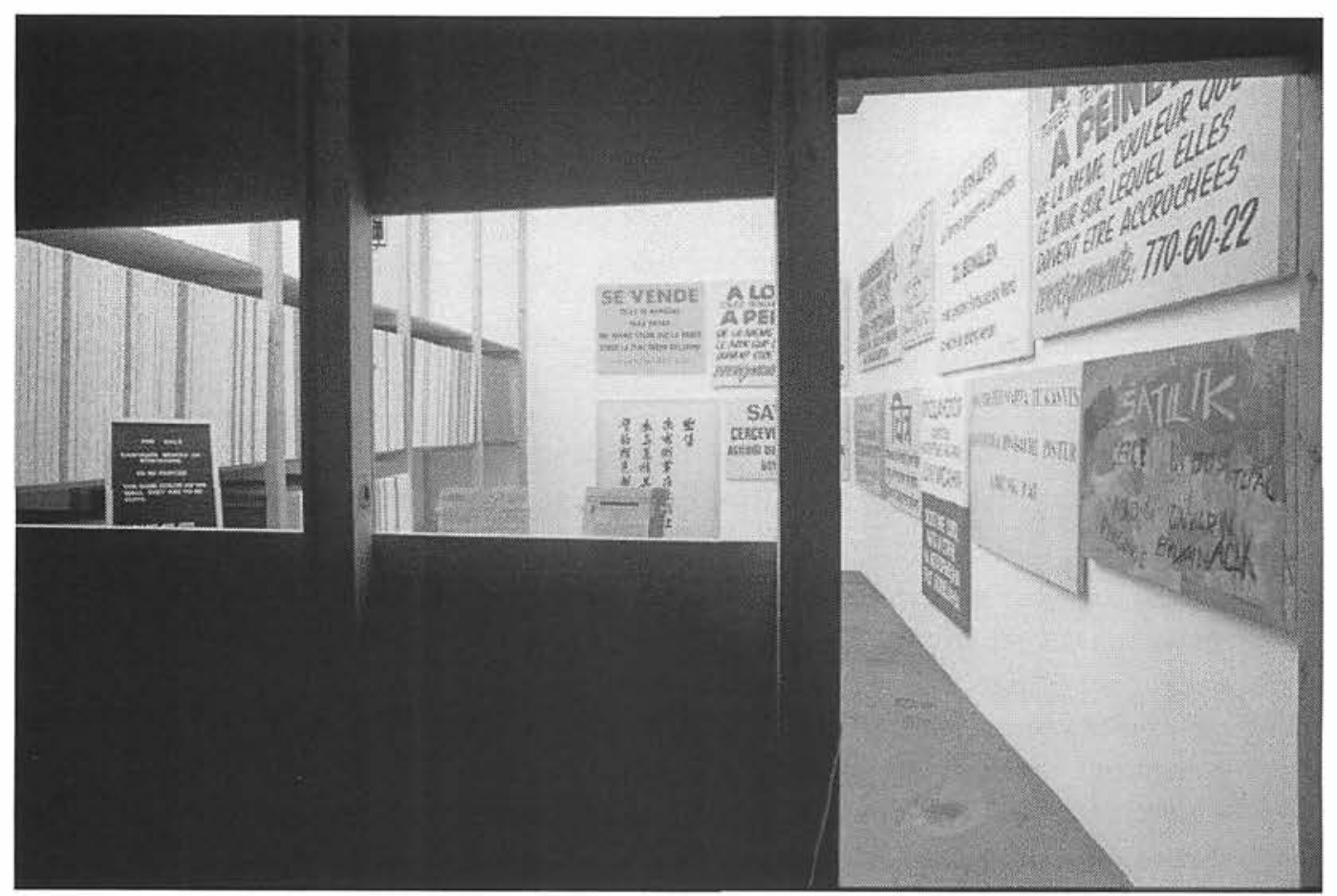

Illustration 6

Claude Rutault, $d / m$ 170 [inventaire 2], 1981, 249 toiles (stock de toiles égal au nombre de définitions/méthodes écrites au jour de l'installation et présentées dans un lieu aménagé pour la d/m 170: un espace fermé, un côté vitré avec des étagères sur lesquelles sont présentées verticalement les 249 toiles), coll. Mamco, ๑ Mamco, Genève. [photo: I. Kalkkinen.] La 176 internationale d/m ,1981-1997, ensemble de 13 panneaux, coll. Jean Brolly, Paris, est actualisée dans l'espace de l'inventaire 2 


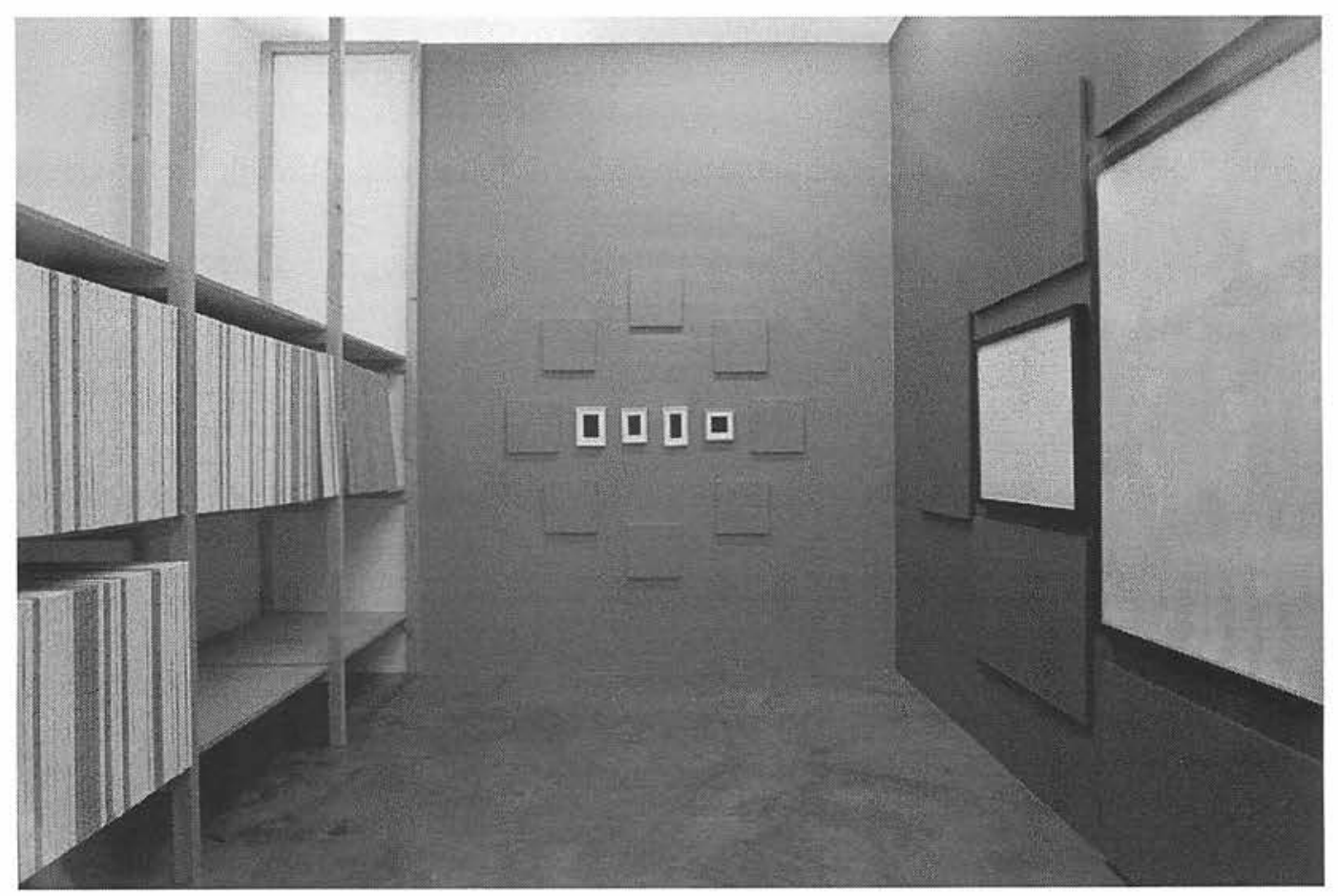

\section{Illustration 7}

Claude Rutault, $d / m 170$ [inventaire 2], 1981, 249 toiles (stock de toiles égal au nombre de définitions/méthodes écrites au jour de l'installation et présentées dans un lieu aménagé pour la d/m 170: un espace fermé, un côté vitré avec des étagères sur lesquelles sont présentées verticalement les 249 toiles), coll. Mamco, oMamco, Genève. [photo: I. Kalkkinen.] La d/m 131 [entourant le tableau], 1981, coll. De l'artiste est actualisée dans l'espace de l'inventaire 2 
30

Un travail similaire est effectué dans le cadre de L'Atelier d'aujourd'hui de Collin-Thiébaut qui reçoit régulièrement des invités. Bernard y intègre des artistes ayant des approches différentes de l'appropriation: Sherrie Levine, Ernest T., Présence Panchounette, Braco Dimitrijevic, André Raffray, etc.

31

MAMCO. Expositions temporaires précédentes, <http://www.mamco. ch/expositions/precedentes/vive6. html> (consulté le 6 juillet 2010). 32 BERNARD, Christian. Conjonctions 7. Mamco Radio. <http://www. mamco.ch/Radio/Conjonctions_7. htm> (consulté le 6 juillet 2010). nouvel éclairage de la démarche de Rutault et procède de la production d'un microrécit de l'art contemporain ${ }^{30}$.

L'actualisation de la $d / m 131$ relève de surcroît d'une reprise et d'une relecture des dispositifs d'exposition mis en œuvre au cours de l'histoire du Mamco. Elle s'inscrit dans une série d'expositions intitulée Répertoire (Replay) $n$, $n$ bis et 12 dans laquelle le "musée rejoue de façon inédite des fragments de certaines de ses expositions réalisées depuis 1994 " ${ }^{31}$. Intitulé Paso doble 2, le trio Rutault, McCollum et Prina constitue une reprise de l'exposition Paso doble que Bernard avait réalisée en 1994 au Musée d'art contemporain de Pully. Elle consistait à confronter dans chacune des salles Rutault à un autre artiste. Les murs dédiés aux actualisations des $\mathrm{d} / \mathrm{m}$ de Rutault étaient peints de couleur, ceux destinés à recevoir les œuvres des autres artistes étaient laissés blancs, "Il s'agissait de créer à chaque fois un 'pas de deux' dont les deux protagonistes pouvaient être très proches ou au contraire très éloignés. " Pour Paso doble 2, Bernard pensait les appariements "en mettant en corrélation des œuvres apparemment dissemblables. Des artistes qui ont établi le fondement de leur travail dans les années 1960 , mais qui ont des trajectoires, des cultures et des problématiques assez différentes. " 32 L'espace monographique de Rutault est donc à la fois une œuvre, un lieu de production de microhistoires de l'art contemporain, un terrain d'expérimentation dans lequel l'institution procède à une révision critique de ses propres stratégies muséologiques.

Le Container zéro de Jean-Pierre Raynaud relève en apparence d'un cas de figure similaire à celui de l'inventaire 2 : il répond à une commande du CNAC et du MNAM passée à l'artiste en 1988 ; il a un statut d'œuvre d'art tout en fonctionnant comme un espace d'exposition ouvert au travail d'autres artistes. L'œuvre consiste en un container dont l'extérieur est peint en blanc et l'intérieur entièrement recouvert du matériau de prédilection de Raynaud, les carreaux de céramique blancs (ill. 8). L'artiste qui assume le rôle de conservateur de cet espace y présente des artefacts de son choix pour une période qu'il détermine. L'œuvre est évolutive et se transforme à chaque nouvel accrochage. 
Des espaces monographiques au sein des collections muséales : déléguer ou produire une pensée et une pratique critiques de la collection?
La mise en exposition d'objets à même un espace, une stèle ou une surface carrelé est un geste récurrent dans la pratique artistique de Raynaud. Le carreau blanc joue le rôle d'un "refroidisseur": "il remet les choses à plat comme un degré zéro des émotions venues du monde extérieur " 33 . L'artiste s'adonnait régulièrement à cette pratique d'exhibition d'objets dans sa maison de La Celle-Saint-Cloud dont l'intérieur était entièrement carrelé. Le Container constitue le prolongement public de cette expérience. Son inauguration en 1988 intervient au moment où Raynaud prend la décision de fermer sa maison au public pour une durée de 20 ans, avant de la détruire en 1993. "Le projet est sorti [de ma maison], dit-il. Et le Container est un peu son testament, son reliquaire, ${ }^{34}$

Un contrat, sous forme de lettre, reconnaît à l'artiste la responsabilité de la programmation de Container et attribue au musée un rôle consultatif, sans que les modalités de la consultation ne soient précisées: "Pour ce qui concerne le Container lui-même, j'ai noté votre accord pour me considérer comme le conservateur de l'espace zéro de ce container. Ainsi, j'aurai de mon vivant la responsabilité de tout accrochage ou présentation à l'intérieur de cet espace, après avis de votre part. " ${ }^{35}$ Le contrat prévoit également qu'un tableau carrelé blanc portant le chiffre zéro sera accroché dans le Container de façon permanente à la mort de l'artiste, arrêtant définitivement l'évolution de l'œuvre.

A l'ouverture de Container, Raynaud a privilégié des œuvres d'autres artistes issues de la collection du MNAM et qui avaient marqué sa démarche artistique: le pigment bleu d'Yves Klein, le Carré noir sur fond blanc de Kasimir Malevitch, une sculpture-machine de Jean Tinguely, etc. "Selon mes désirs, explique Raynaud, ce lieu doit pouvoir accueillir une cuvre du musée comme expérience personnelle, être fermée, être révélée d'une autre façon, non prévue encore. " ${ }^{36}$ Avec le temps, Raynaud semble de moins en moins ouvert à exposer d'autres artistes et le Container tend à devenir un espace consacré à sa propre œuvre, voire à sa vie personnelle. Il y dépose des œuvres représentatives de ses recherches plastiques du moment ou de ses expositions
33 Jean-Pierre Raynaud, en entretien avec Catherine Lawless. "JeanPierre Raynaud, Container zéron. Les Cahiers du Musée national d'art moderne, $n^{2} 23$, printemps 1988 ,

p. 120.

34

Id., p. 118.

35

Lettre de Jean-Pierre Raynaud à Jean-Hubert Martin qui tient lieu d'entente entre l'artiste et l'institution, septembre 1988. Dossier de l'œuvre. Documentation des Collections contemporaines, Musée national d'art moderne, Paris.

36

Jean-Pierre Raynaud, en entretien avec Catherine Lawless, op. cit., p. 119. 


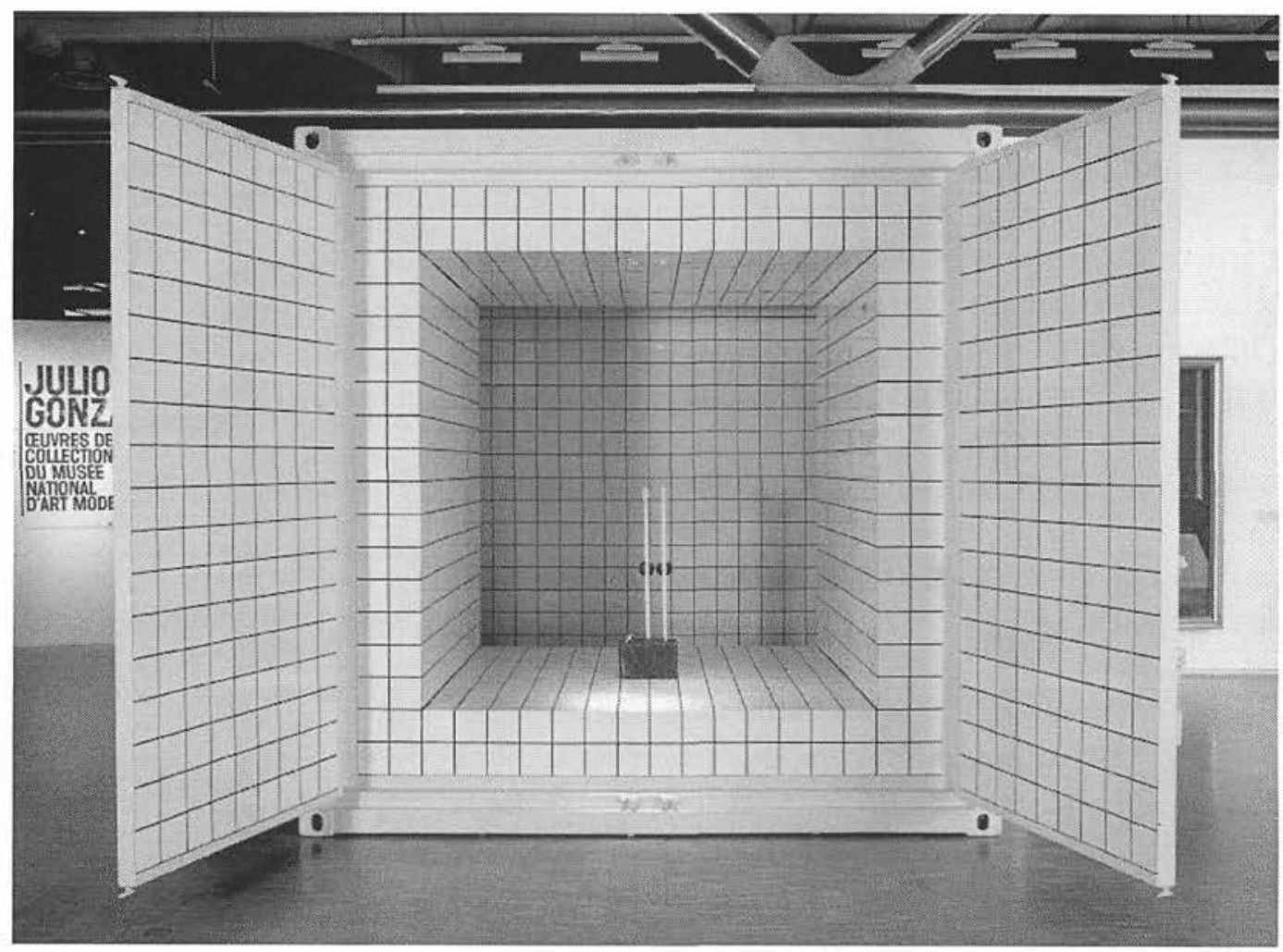

Illustration 8

Jean-Pierre Raynaud, Container Zéro, 1988, acier et carreaux de céramique, 330 × 330 × $330 \mathrm{~cm}$, Musée National d'Art Moderne, Centre Pompidou, Paris, France, AM1988-2(1), $\odot$ CNAC/ MNAM/Dist. Réunion des Musées Nationaux/Art Resource 
Des espaces monographiques au sein des collections muséales: déléguer ou produire une pensée et une pratique critiques de la collection? importantes et des objets témoignant des événements marquants de sa vie personnelle. Le Container dresse une représentation synthétique de la démarche artistique et de la vie de Raynaud qui se déploie sur un plan diachronique. Sur le plan pragmatique, l'artiste consulte de moins en moins les conservateurs et réalise parfois de nouveaux accrochages sans les prévenir. Il engage son propre photographe pour documenter les occurrences successives de l'œuvre. Ce glissement vers une gestion individuelle entraîne un certain nombre de tensions.

Les échanges de courriels entre la conservatrice et le directeur du MNAM et les comptes rendus des discussions qu'ils ont eues avec Raynaud permettent de mieux saisir le différend entre l'artiste et le musée ${ }^{37}$. Les professionnels du musée souhaitent que les accrochages réalisés dans le Container établissent un dialogue avec les autres expositions. A plusieurs reprises, ils ont demandé à Raynaud de modifier le contenu de l'œuvre pour l'ouverture d'une nouvelle exposition. Ils l'ont également incité à continuer d'y exposer d'autres artistes. Régulièrement, ils lui ont rappelé le souhait du MNAM d'être davantage intégré au processus, selon un mode plus collaboratif, refusant de s'en tenir au strict rôle de dépositaire de l'œuvre. Il semble que, pour les professionnels du MNAM, le Container, tout en relevant d'un dispositif d'exposition entièrement subjectif, devrait permettre une relecture de certaines œuvres de la collection, voire de certaines expositions, afin d'établir un rapport d'intertextualité avec l'espace du musée et son contenu. Raynaud, au contraire, conçoit le Container selon une logique d'isolement par rapport à l'espace du Centre Pompidou et d'indépendance à l'égard du musée. De plus, l'artiste a toujours revendiqué l'enfermement, la solitude, la subjectivité au cœur de sa démarche artistique, valeurs qu'il réitère pour la programmation du Container. Le différend relève donc de divergences de conceptions difficiles à concilier.

Les espaces monographiques de BGL, de Cérard CollinThiébaut et de Claude Rutault se distinguent des premiers par les modalités selon lesquelles ils s'inscrivent dans les

\section{7}

Courriers électroniques échangés entre Nadine Pouillon et Alfred Pacquement, surtout au cours des années 2001 et 2002. Dossier de l'œuvre. Documentation des Collections contemporaines, Musée national d'art moderne, Paris. 
institutions qui les collectionnent. Leur portée critique ne tient pas seulement aux conceptions de la collection que les artistes y formalisent, mais surtout aux modes d'interaction qu'ils établissent avec les professionnels du musée. Ces œuvres les sollicitent à s'interroger sur leur pratique de collection, voire à la redéfinir. La capacité de ces espaces monographiques à générer de nouvelles pratiques muséales repose en partie sur le partage de l'autorité auctoriale de l'artiste qui prend des formes très différentes, mais qui semble corrélatif à l'ambiguité du statut de l'espace monographique. L'espace monographique de BCL fonctionne selon un double régime. Ou bien il prend la forme d'une installation sur laquelle le collectif exerce sa pleine autorité d'auteur, ou bien il se présente comme un corpus imposant d'œuvres autonomes que la conservatrice du musée devra faire vivre au sein de la collection. L'espace monographique de Collin-Thiébaut, dont le statut oscille entre la scénographie d'exposition et l'installation, est le fruit d'une cocréation par l'artiste et le directeur du musée, dans le respect des champs de compétences artistique et muséologique des acteurs respectifs. Selon un processus de délégation et de responsabilisation, le musée, en tant que preneur en charge de l'inventaire 2, non seulement réalise, actualise, diffuse et documente l'œuvre de Rutault, mais la travaille comme un espace d'exposition dans lequel les professionnels du musée exercent pleinement leur autorité auctoriale dans le respect des prérogatives de l'artiste. Chez Raynaud, bien qu'il soit aussi conçu comme un lieu d'exposition et qu'il réponde à une volonté de la part des professionnels du musée de générer une réflexion sur la collection et ses modes de présentation, l'espace monographique ne parvient pas à établir des formes de collaboration, l'artiste accomplissant une individualisation et un repliement de son espace.

La réussite de ces expériences collaboratives semble reposer largement sur les cultures institutionnelles qui doivent privilégier le dialogue avec les artistes et inciter les professionnels du musée à assumer pleinement un travail d'interprétation des œuvres. Au Mamco, jeune institution créée en 1994, l'espace monographique tient une place singulière, car son directeur, très sensible à la réticence des artistes 
à l'égard du collectionnement échantillonnant, en a fait l'un des axes principaux de développement de la collection. La singularité de son approche tient du fait que ces espaces monographiques sont élaborés en étroite collaboration avec les artistes et reprennent plusieurs des formes et des dispositifs muséaux que les artistes ont imaginés depuis le milieu du XIX siècle pour conserver et diffuser leur propre œuvre ou celle des autres, en retrait des modèles institutionnels traditionnels. De plus, au Mamco, la collection et l'exposition étant étroitement articulées, les espaces monographiques sont d'emblée pensés dans la perspective de leur mise en exposition, de leurs réactualisations et des multiples microrécits de l'art contemporain qu'ils peuvent intégrer et engendrer. Leur essoufflement est également anticipé, l'institution s'octroyant la possibilité de reformuler leur mode de fonctionnement, voire de mettre un terme à leur existence, en accord bien sûr avec les artistes.

La collection d'art contemporain du MBAC, à l'instar du MNAM, combine une double approche de la collection, procédant par échantillonnage et par séries monographiques ${ }^{38}$. Elle détient donc d'importants corpus de certaines figures artistiques internationales, nationales et régionales, que les conservateurs successifs ont ciblées et avec lesquelles ils ont entretenu des relations étroites, propices à des modes de travail collaboratifs. L'espace monographique ne constitue toutefois pas un axe de la collection et l'acquisition du Discours des éléments présente à cet égard un caractère exceptionnel. L'échantillonnage auquel procède l'institution ne suit pas les canons du modernisme. Dans la foulée des débats sur le postmodernisme, le MBAC a tenté d'articuler au sein de sa collection une représentation des différentes régions canadiennes, une reconnaissance de la diversité des communautés et des groupes minoritaires, en l'occurrence les artistes autochtones et les artistes femmes. Ce syncrétisme exige de développer une capacité à produire des récits de l'art contemporain multiples, fragmentés, contradictoires, favorisant parmi les professionnels de l'institution une réflexion critique sur la production discursive au sein de la collection. Enfin, au cours des dernières années, l'institution a développé une
38

LEVESQUE, op. cit., p. 145-209. 
39

BAL, Mieke. "Le public n'existe pas". In. CAILLET, Elisabeth et Catherine Perret (dir.). L'art contemporain et son exposition (2). Paris: L'Harmattan, 2007, p. 9-35 expertise en matière de conservation et de restauration de l'art contemporain qui s'est accompagnée d'une réévaluation des valeurs traditionnelles de pérennité et d'authenticité et d'une réflexion sur la réexposition et la réactualisation des œuvres. L'argumentaire de Josée Drouin-Brisebois en faveur de l'acquisition de l'œuvre de BCL repose sur ces deux axes de réflexion.

\section{Un phénomène d'individuation?}

L'acquisition d'espaces monographiques offre des réponses pertinentes aux limites des modèles traditionnels de collection. Elle permet de privilégier des formes holistiques qui rendent mieux compte de la démarche de l'artiste que quelques échantillons. Elle tend à réhabiliter la valeur artistique des œuvres qui est traditionnellement secondarisée au profit de leur valeur historique. Elle engendre souvent des processus de réactualisation qui favorisent un renouvellement de l'interprétation des œuvres. Elle instaure parfois des formes de collaboration entre les artistes et les professionnels de musées qui amènent ces derniers à aborder leur pratique de façon plus critique. Enfin, elle permet de réhabiliter une généalogie de formes de pérennisation et de mise en exposition des œuvres que les artistes ont imaginées depuis le $\mathrm{XIX}^{\mathrm{e}}$ siècle et qui ont été exclues jusqu'à très récemment de l'histoire de l'art et de la culture muséale.

Mais l'intégration des espaces monographiques aux collections muséales relève également d'un phénomène d'individuation qui peut soulever un certain nombre d'interrogations. Les espaces monographiques pour lesquels les artistes assument l'entière responsabilité de leur implémentation et de leurs variations selon un processus de délégation du musée à l'artiste ne procèdent-ils pas d'une survalorisation des intentions de l'artiste? Le musée se tient dans une position de retrait qui reconduit la pseudo neutralité du «cube blanc» dans la perspective quelque peu illusoire de transmettre au public l'intention de l'artiste sans "interférence", de la façon la plus directe possible ${ }^{39}$. Corollairement, on peut se demander si les formes plus collaboratives d'espaces monographiques ne procèdent pas d'une 
trop forte "auteurisation" du conservateur, surtout lorsque celui-ci les co-crée avec l'artiste ou qu'il prend l'initiative d'une variation qui a d'importantes incidences sur le plan formel ou sémantique. Il me semble qu'un espace monographique collaboratif dépasse ce phénomène d'individuation dans la mesure où il contribue à produire des microrécits de l'art contemporain et qu'il génère donc de nouvelles lectures et interprétations des œuvres. Cela suppose que dans cette production discursive le conservateur assume pleinement son rôle d'énonciateur. 


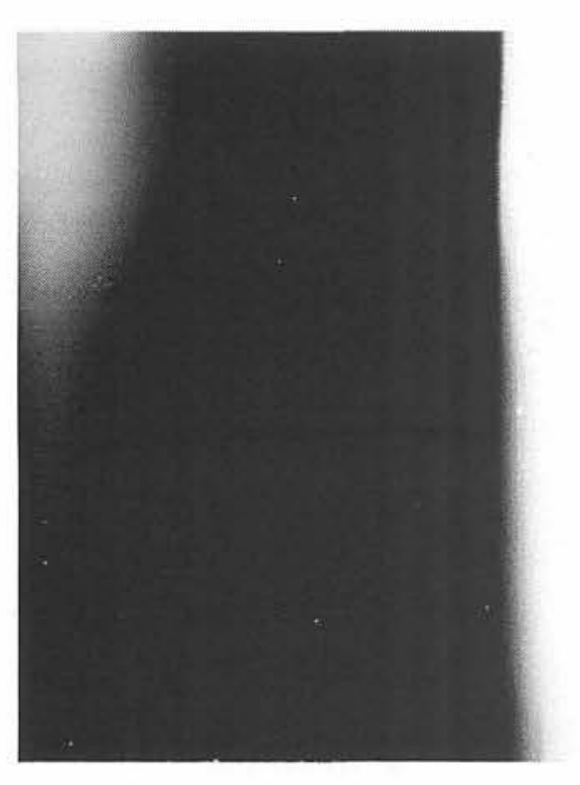

\author{
Summary \\ Translated by Philippe Lagrange \\ Monographic Spaces \\ Within Museum Collections: \\ Delegating or Producing a \\ Critical Thought and Practice \\ of the Collection.
}

This article studies the monographic spaces dedicated to some artists within museum collections. These art objects, which first appeared in the late 1960's, overcome in many aspects the common conception of a work of art forming a synthesis out of many works that had previously been conceived and often presented separately. It is frequent to see these monographic spaces evolve, their acquisition by a museum not necessarily forbidding their modification and even sometimes allowing to prepare and to manage that transformation. Finally, these monographic spaces are recognizable by their "exposed" or "installative" character, the spectator being uncertain whether he should perceive them as expositions or installations and this ambiguity deepens when a monographic space displays the work of other creators.

The aim of this study is to evaluate whether or not the collection of monographic spaces solves some of the modern art museum's aporias that have been widely and regularly decried by artists since the creation of the museums: the sample collecting method, in which an artwork is considered the synecdoche of the artist's whole practice or of an important period of his evolution; the tendency to temporally fix the artwork at the moment of its acquisition, valorising the "historical worth" at the expense of the "artistic value" (F. Choay); 
and the staging of the permanent collections which are too often be limited by historical tales, chronological, stylistical or national considerations, which are deemed obsolete, especially since the debates on post-modernism.

This essay examines seven cases studies chosen for the exemplarity of their form and their monographic space status; for the way they were acquired as for the collecting methods of the acquiring institutions. The analysis shows that collecting monographic spaces provides valuable solutions to the limits of the traditional collection patterns. It allows to favour holistic forms of collection giving a better understanding of the artist's approach than the sampling of a few works. Also, it tends to rehabilitate the "artistic value" of the artworks as it often induces reactualisation processes who advocate for a reactualisation of their interpretation. Sometimes, it initiates collaboration between the artists and the museum professionals, thus pushing the artists to look at their practice with a more critical eye. Finally, these modes of collecting allow a rehabilitation of a genealogy of perpetuation forms and of artwork exhibition imagined by artists since the $X I X^{\text {th }}$ century that have been excluded from art history and museum culture until recently.

The analysis also permitted to distinguish two different institutional point of view. For certain museums, the acquisition of monographic spaces allows the integration of some critical forms of collecting without the obligation of producing them nor having to put them in application according to a principle of delegation to the artist and the folding back of the monographic space within the reach of the artwork itself. Among others, we remark some forms of collaboration, co-production, and sometimes, an appropriation of certain strategies used by the artists in their monographic spaces. Museum professionals do not only delegate the production of a critical form of collection to the artist but they also contribute to its making by collaborating with him. In these cases, the monographic space is conceived as an artistic and museological experimental space elaborated by both the artist and the institution, therefore generating new forms of artwork perpetuation and contemporary art heterodox tales. 